Elsevier required licence: () 2018.

This manuscript version is made available under the CC-BY-NC-ND 4.0 license http://creativecommons.org/licenses/by-nc-nd/4.0/

The definitive publisher version is available online at 10.1016/j.neucom.2018.07.017 


\section{Accepted Manuscript}

Consensus of multi-agent systems with faults and mismatches under switched topologies using a delta operator method

Dianhao Zheng, Hongbin Zhang, J. Andrew Zhang, Gang Wang

PII: S0925-2312(18)30845-2

DOI: 10.1016/j.neucom.2018.07.017

Reference: NEUCOM 19765

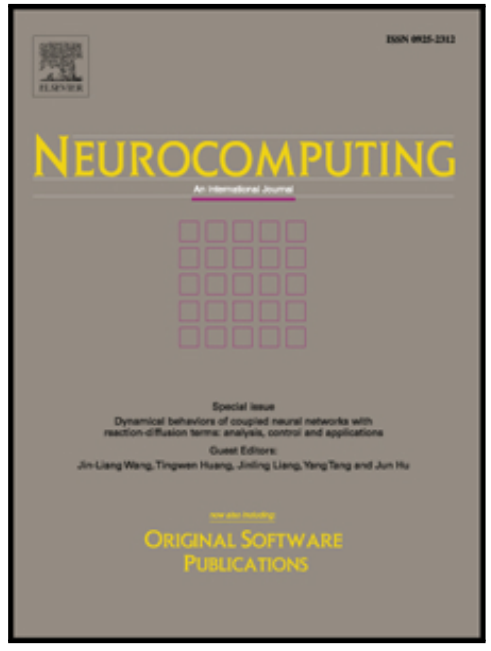

To appear in:

Neurocomputing

Received date:

7 December 2017

Revised date:

22 May 2018

Accepted date:

1 July 2018

Please cite this article as: Dianhao Zheng, Hongbin Zhang, J. Andrew Zhang, Gang Wang, Consensus of multi-agent systems with faults and mismatches under switched topologies using a delta operator method, Neurocomputing (2018), doi: 10.1016/j.neucom.2018.07.017

This is a PDF file of an unedited manuscript that has been accepted for publication. As a service to our customers we are providing this early version of the manuscript. The manuscript will undergo copyediting, typesetting, and review of the resulting proof before it is published in its final form. Please note that during the production process errors may be discovered which could affect the content, and all legal disclaimers that apply to the journal pertain. 


\title{
Consensus of multi-agent systems with faults and mismatches under switched topologies using a delta operator method ${ }^{\underline{\pi}}$
}

\author{
Dianhao Zheng ${ }^{\mathrm{a}, \mathrm{b}}$, Hongbin Zhang ${ }^{\mathrm{a}, *}$, J. Andrew Zhang ${ }^{\mathrm{c}}$, Gang Wang ${ }^{\mathrm{a}}$ \\ ${ }^{a}$ School of Information and Communication Engineering, University of Electronic Science and Technology of China, Chengdu, Sichuan, 611731, \\ China. \\ ${ }^{b}$ Faculty of Engineering and Information Technology, University of Technology Sydney, Sydney 2007, Australia. \\ ${ }^{c}$ Global Big Data Technologies Centre, University of Technology Sydney, Sydney 2007, Australia.
}

\begin{abstract}
This paper studies the consensus of multi-agent systems with faults and mismatches under switched topologies using a delta operator method. Since faults and mismatches can result in failure of the consensus even for a fixed topology with a spanning tree, how to reach a consensus is a complicated and challenging problem under such circumstances especially when part topologies have no spanning tree. Although some works studied the influence of faults and mismatches on the consensus, there is little work on reaching a consensus for the multi-agent systems with faults and mismatches. In this paper, we introduce the delta operator to unify the consensus analysis for continuous, discrete, or sampled systems under one framework. We develop the theories on the delta operator systems first and then apply theories of the delta operator systems to the consensus problems. By converting the consensus problems into stability problems, we investigate and prove consensus and the associated conditions for systems 1) without any fault, 2) with a known fault, and 3) with unknown faults, under switching topologies with matching or mismatching coefficients.
\end{abstract} Numerical examples are provided and validate the effectiveness of the theoretical results.

Keywords: delta operator system, switched topologies, faults and mismatches, consensus, multi-agent sytems

\section{Introduction}

Collective behaviors widely exist in nature such as flocking of birds and schooling of fish. They have attracted more and more attention. Collective behaviors of groups rely on local interactions among individuals, which drives the emergence of coordination at the group scale [1]. Although the general mechanisms of coordination are not completely understood, known underlying mechanisms, for example, globally coordinated behaviors arise from local interactions, have helped us better understand the cooperative control of multi-agent systems [1, 2]. Coordinations have received increasing interests for their broad applications [3-6].

\footnotetext{
${ }^{*}$ Corresponding author

Email address: zhanghb@uestc.edu.cn (Hongbin Zhang)

URL: dianhao18@126. com (Dianhao Zheng), Andrew. Zhang@uts . edu . au (J. Andrew Zhang), wanggang_hld@uestc.edu.cn (Gang Wang)
} 
The consensus, as one of the most fundamental collective behaviors, refers to reaching a common state for all initial conditions. This common state is not decided by centralized systems but by distributed systems with the local information of every agent and its neighbors [7,8]. The consensus problem has a long history, and it has received considerable attention in the last two decades [8-10]. The consensus problem under ideal conditions is well studied, and there is also limited work studying the influence of faults and mismatches on consensus. Reaching consensus in the presence of faults and mismatches is very challenging as they can result in failure of the consensus even for a fixed topology with a spanning tree, particularly when the system is continuous, discrete or sampled.

Consensus with mismatching coefficients is of practical importance. Some preliminary results on the consensus are reported in [11-18]. According to the survey [11], consensus can be reached if the undirected network digraph is strongly connected. Because the undirected networks can be treated as special cases of directed networks where the adjacency matrices are symmetric, the conditions of directed networks are stricter than those of undirected networks. The article [12] reports that the first-order consensus can be achieved asymptotically if the union of the directed interaction graphs has a spanning tree sufficiently frequently as the system evolyes. It is shown in [13] that the second-order consensus requires other conditions in addition to having a spanning tree. The work [17] states that a suitable Laplacian matrix and proper coupling strengths are indispensable to achieve consensus for second-order systems under fixed topologies. That is, the second-order systems cannot reach consensus with improper strength coefficients even for fixed topologies with a spanning tree. These studies and results indicate that achieving consensus with mismatching coefficients is important and useful.

Mismatches resulting in failure of the consensus under fixed topologies does not necessary mean a failure under switched topologies. There is limited research on the consensus for topologies with mismatches and faults, considering varying topologies. In [15-18], conditions for the consensus are analysed with reference to the choice of coefficients under fixed topologies. But these papers did not explain when and how these topologies switch with the proper or improper coefficients.

With the development of cooperative control, multi-agent systems become increasingly complex, and faults cause severer impacts on system performance [19]. The sources of faults are multifarious, such as external measurements, internal settings and weighted deviations. These faults can cause system performance deterioration and lead to instability which can further result in catastrophic accidents. Thus, many effective fault tolerant control approaches have been proposed to improve system reliability [19-23]. The authors in [19] studied cooperative adaptive fuzzy tracking control for a network with unknown actuator faults which are limited to a weighted directed graph with a fixed topology. The paper [20] presents a cooperative actuator fault accommodation strategy for a team of multi-agent systems with a switching topology on the assumption that the digraph is always strongly connected. The paper [21] presents an adaptive fault-tolerant control scheme for leader-follower consensus control of uncertain mobile agents with actuator faults under a fixed topology. The study in [22] addresses the cooperative fault-tolerant tracking control problem for a class of multi-agent systems subject to mismatched parameter uncertainties, external disturbances and actuator faults under undirected connected graph with a fixed topology. The authors in [23] propose an $H_{\infty}$ cooperative fault recovery 
control scheme confined to the fixed topology with a spanning tree. In actuality, a consensus is likely reached under switched topologies with unknown faults and a lack of the spanning tree. However, detailed realization is unknown yet.

With the wide use of computers in modern control engineering and the indispensable sampling operation to accomplish computerized processing, the sampled multi-agent systems have been studied in recent year[2, 8,9]. This situation can also be found in the signal processing field[24, 25]. These works separately study discrete or sampled multi-agent systems from continuous ones, which are effective for individual systems, but lack of generality. It is also hard to bridge and connect between continuous and sampled systems. Therefore, finding a suitable tool to unify the continuous and sampled multi-agent systems is very important. Based on the work on the delta operator [26-31], the delta operator is compatible with continuous and discrete time systems, and hence can be a good option for such a unifying tool.

This paper aims to study the consensus of multi-agent systems with faults and mismatches under switched topologies using a delta operator method, to address the major limitations of current research as mentioned above. The main contributions of this paper are as follows:

- We develop the method on the delta switched systems and this method unify consensus analysis for continuous, discrete and sampled systems under one framework;

- We investigate and prove the consensus and its associated conditions for systems with no fault, a known fault, and unknown faults originating from various sources; and

- We analyse consensus under switching topologies with matching and mismatching coefficients in every case, and demonstrate the associated conditions of consensus;

Our developed theorems in this paper have no special limitations. The topologies can be undirected or directed and the network can be balanced or unbalanced. The results in this paper can be applied, but not limited, to the following cases: no leader; switching leaders; switching between with and without a leader.

The rest of this paper are organized as follows: some basic concepts are provided in Section2. Section 3 presents the main results for three cases of the multi-agent systems. Numerical examples are made in Section 4 to verify the theoretical analysis. Finally, the conclusion is given in Section 5.

Notations: $X^{T}$ denotes the transpose of a matrix $X, P>(<) 0$ denotes that the matrix $P$ is symmetric and positive (negative) defínite, $I$ represents an identity matrix, $C^{1}$ represents the space of continuously differentiable functions, and $\mathbb{R}^{N}$ denotes the $N$-dimensional Euclidean space.

\section{PRELIMINARIES}

In this section, some important basic graph theories are introduced. 
A multi-agent system with $n$ agents is considered in this paper. Basic graph theory is used to model the undirected or directed interaction among agents. The set of node indexes is $\mathcal{I}=\{1,2, \ldots, n\}$. Let a directed graph $\mathcal{G}=(\mathcal{V}, \mathcal{E}, \mathcal{A})$ describe the communication topology among the agents, where $\mathcal{V}=\left\{v_{1}, v_{2}, \ldots, v_{n}\right\}, \mathcal{E} \subseteq \mathcal{V} \times \mathcal{V}$, and $\mathcal{A}=\left[a_{i j}\right]$. They are the set of nodes, a set of edges, and the corresponding adjacency matrix, respectively. We assume that there are no self loops in the networks. The set $\mathcal{N}_{i}=\left\{v_{j} \in \mathcal{V}:\left(v_{i}, v_{j}\right) \in \mathcal{E}, j \neq i, j \in \mathcal{I}, i \in \mathcal{I}\right\}$ stands for the set of neighboring node $v_{i}$. The matrix $L=\left[l_{i j}\right]$ is called as a Laplacian matrix, where 1) $l_{i j}=\sum_{k=1, k \neq i}^{n} a_{i k}$ for $\mathrm{i}=\mathrm{j}$; and 2$) l_{i j}=-a_{i j}$, for $i \neq j$, and $i, j \in \mathcal{I}$. A directed graph is called as a directed tree, when every node has exactly one parent except for the root. A directed tree is seen as a spanning tree of a directed graph when the tree connects all the nodes of the graph. More details can be found in [32]. A new matrix $H$ is defined as $H=\left[h_{i j}\right] \in \mathbb{R}^{(n-1) \times(n-1)}$, where $h_{i j}=l_{i j}-l_{n j}$.

For the switched topologies, we assume that there are $m$ topologies, where $m$ is a positive constant. A finite set $\mathcal{J}=\{1,2, \ldots, m\}$ denotes all the switched topologies.

For the considered multi-agent system with $n$ agents, every agent is modeled using the $G^{\text {th }}$-order model [17, 3335]:

$$
\begin{gathered}
\dot{x}_{i}^{(0)}(t)=x_{i}^{(1)}(t) \\
\vdots \\
\dot{x}_{i}^{(G-2)}(t)=x_{i}^{(G-1)}(t) \\
\dot{x}_{i}^{(G-1)}(t)=u_{i}, i \in \mathcal{I} .
\end{gathered}
$$

where $x_{i}(t)$ is the state of the $i^{t h}$ agent, $u_{i}$ is the control input, and $x_{i}^{(k)}$ stands for the $k^{t h}$ derivative of $x_{i}$ and $x_{i}^{(0)}=x_{i}$. Since it is harder to get higher order information of other agents in many circumstances, we consider the following consensus algorithm[33, 34]:

$$
u_{i}(t)=\beta_{0} \sum_{j \in \mathcal{N}_{i}(t)} a_{i j}(t)\left(x_{j}^{(0)}(t)-x_{i}^{(0)}(t)\right)-\beta_{1} x_{i}^{(1)}(t)-\cdots-\beta_{G-1} x_{i}^{(G-1)}(t), i=\mathcal{I},
$$

where the positive constants $\beta_{0}, \beta_{1}, \beta_{2}, \ldots, \beta_{G-1}$ denote the (coupling or strength) coefficients, and $a_{i j}, i, j \in \mathcal{I}$, are the entries of the adjacency matrix $\mathcal{A}(\mathcal{G})$ for the given interaction topology $\mathcal{G}$. Let $\mathcal{K}$ denote the set of $\{0, \ldots, G-1\}$.

Remark 1. The methods and results in this paper are applicable to other consensus algorithms, such as the consensus algorithm on full coupling state information [17, 35]:

$$
u_{j}(t)=\beta_{0} \sum_{j \in \mathcal{N}_{\mathrm{i}}(\mathrm{t})} a_{i j}(t)\left(x_{j}^{(0)}(t)-x_{i}^{(0)}(t)\right)+\cdots+\beta_{G-1} \sum_{j \in \mathcal{N}_{\mathrm{i}}(\mathrm{t})} a_{i j}(t)\left(x_{j}^{(G-1)}(t)-x_{i}^{(G-1)}(t)\right) .
$$

Only the value of $\hat{A}, \hat{I}$ and $\hat{B}$ in this paper need to be revised accordingly.

Remark 2. For multi-agent systems with proper consensus algorithms, different topologies will cause different $a_{i j}$. Correspondingly, a fixed topology means an invariable value of $a_{i j}$.

Definition 1. The $G^{\text {th }}$-order consensus of the multi-agent systems with $n$ agents is said to be reached only if $x_{i}^{(k)}(t) \rightarrow$ $x_{j}^{(k)}(t), k \in \mathcal{K}$, when $t \rightarrow+\infty$, for all initial conditions. 
Definition 2. [36] For a switched system with the switching signal $\sigma(t)$ and any $T>t \geq 0$, the notation $N_{\sigma p}(T, t)$ stands for the switching numbers of the $p^{\text {th }}$ subsystem, and $T_{p}(T, t)$ denotes the total running time of $p^{\text {th }}$ subsystem over the interval $[t, T)$. The system is said to have a mode-dependent average dwell time (MDADT) $\tau_{a p}$ if positive numbers $N_{0 p}(T, t)$ and $\tau_{a p}$ exist, and such that

$$
N_{\sigma p}(T, t) \leq N_{0 p}(T, t)+\frac{T_{p}(T, t)}{\tau_{a p}} .
$$

Consider the following delta operator switched systems :

$$
\delta z(t)=f_{\sigma}(z(t)), z\left(t_{0}\right)=z_{0}, t \geq t_{0},
$$

where $z(t)$ and $\sigma$ are the state vector and the switching signal, respectively. The delta operator is defined as

$$
\begin{gathered}
\delta=\left\{\begin{array}{cc}
\frac{d}{d t}, & T=0, \\
\frac{q-1}{T}, & T \neq 0,
\end{array}\right. \\
\delta z(t)=\left\{\begin{array}{cc}
\frac{d z(t)}{d t}, & T=0, \\
\frac{z(t+T)-z(t)}{T}, & T \neq 0,
\end{array}\right.
\end{gathered}
$$

In the above equations, the symbol $q$ denotes the shift operator, $T$ is the sample period. It is shown in [37] that the delta operator system approach the continuous system when the sampling time is sufficiently small. The close connections between this formulation and continuous-time systems are also verified in [37].

Remark 3. For sampled systems, a new topology becomes valid after time $t_{k}$ if the switching occurs during the sampling time $t_{k-1}$ and $t_{k}$ and the new topology sustains long enough. It could be denoted by $\sigma\left(t_{k}\right)=p, \sigma\left(t_{k}^{-}\right)=q$, where $p, q$ stand for two topologies.

Lemma 1. [36] Given a switched system $z(t)=f_{\sigma(t)}(z(t))$, and constants $\lambda_{p}>0, \mu_{p}>1, p \in \mathcal{J}$. If there exist $C^{1}$ functions $V_{\sigma(t)}$, and class $K_{\infty}$ functions $k_{1 p}$ and $k_{2 p}$, such that $\forall p \in \mathcal{J}$,

$$
\begin{gathered}
k_{1 p}(\|z(t)\|) \leq V_{p}(\|z(t)\|) \leq k_{2 p}(\|z(t)\|), \\
\dot{V}_{p}(\|z(t)\|) \leq-\lambda_{p} V_{p}(\|z(t)\|),
\end{gathered}
$$

and $\forall\left(\sigma\left(t_{i}\right)=p, \sigma\left(t_{i}^{-}\right)=q\right) \in \mathcal{J} \times \mathcal{J}, p \neq q$

$$
V_{p}\left(z\left(t_{i}\right)\right) \leq \mu_{p} V_{q}\left(z\left(t_{i}^{-}\right)\right)
$$

then this system is globally uniformly asymptotically stable with MDADT

$$
\tau_{a p} \geq \tau_{a p}^{*}=\frac{\ln \mu_{p}}{\lambda_{p}} .
$$


Lemma 2. [27] For any $p \in \mathcal{J}$, if there exist positive scalars $\lambda_{p} \in(0,1 / T)$, and $T \neq 0, \mu_{p}>1$, a positive definite function $V_{\sigma(t)}$, and class $K_{\infty}$ functions $k_{1 p}$ and $k_{2 p}$, for the delta switched systems (5), such that

$$
\begin{gathered}
k_{1 p}(\|z(t)\|) \leq V_{p}(\|z(t)\|) \leq k_{2 p}(\|z(t)\|), \\
\delta V_{p}(z(t)) \leq-\lambda_{p} V_{p}(z(t)),
\end{gathered}
$$

and $\forall\left(\sigma\left(t_{i}\right)=p, \sigma\left(t_{i}^{-}\right)=q\right) \in \mathcal{J} \times \mathcal{J}, p \neq q$

$$
V_{p}\left(z\left(t_{i}\right)\right) \leq \mu_{p} V_{q}\left(z\left(t_{i}^{-}\right)\right)
$$

then the system (5) is asymptotically stable for any switching signal with MDADT

$$
\tau_{a p}>\tau_{a p}^{*} \triangleq-T \frac{\ln \mu_{p}}{\ln \left(1-\lambda_{p} T\right)} .
$$

Lemma 3. [38] Given matrices $Q, H, E$ and $R$ with appropriate dimensions, $Q=Q^{T}, R=R^{T}>0$. The inequality

$$
Q+H F E+E^{T} F^{T} H^{T}<0
$$

holds for all $F$ satisfying $F^{T} F \leq R$, if and only if there exists some $\varepsilon>0$, such that

$$
Q+\varepsilon H H^{T}+\varepsilon^{-1} E^{T} R E<0 .
$$

\section{MAIN RESULTS}

In this section, we study the consensus of multi-agent systems using a delta method for three cases: systems without any faults, systems with a known fault, and systems with (many types of) unknown faults. In every case, we discuss two situations: (1) switching topologies with matching coefficients; and (2)switching topologies with mismatching coefficients.

\subsection{Case 1: Systems without any fault}

It is proven in $[13,17]$ that improper coefficients can result in the divergence of the state even for a fixed topology with a spanning tree. We/call these topologies (or systems) with improper coefficients as topologies (or systems) with mismatches. For multi-agent systems reaching a consensus under switching topologies, at least two situations could happen: (A) all the topologies are with proper coefficients; (B) Only part topologies are with proper coefficients. For each situation, the multi-agent systems can be continuous, discrete, or sampled. If we analyze all the combinations of situations and systems individually, it would be very complicated.

Alternatively, we introduce the delta operator which generalizes both continuous, discrete or sampled systems. We can then convert the original consensus problems into stability problems in delta switched systems. The above two situations then correspond to two situations in delta systems: (A1) all the subsystems are stable; and (B1) some subsystems are unstable. We can then study the consensus problem through the delta operator switched systems. 


\subsubsection{Stability analysis of the delta operator switched systems}

Firstly, we consider the situation (A1) that all the subsystems are stable in the delta operator switched systems. In this situation, the value of $\sigma$ in the system (5) is from the finite set $\mathcal{J}=\{1, \ldots, m\}$, where $m$ is the number of stable subsystems.

We have the following lemma:

Lemma 4. Consider delta operator switched systems (5) with given positive scalars $\lambda_{p} \in(0,1 / T)$ when $T \neq 0$, or $\lambda_{p}>0$ when $T=0, \mu_{p}>1$. Suppose that there exist $C^{1}$ functions $V_{\sigma(t)}$, and class $K_{\infty}$ functions $k_{1 p}$ and $k_{2 p}$, such that

$$
\begin{gathered}
k_{1 p}(\|z(t)\|) \leq V_{p}(\|z(t)\|) \leq k_{2 p}(\|z(t)\|), \\
\delta V_{p}(z(t)) \leq-\lambda_{p} V_{p}(z(t)),
\end{gathered}
$$

and $\forall\left(\sigma\left(t_{i}\right)=p, \sigma\left(t_{i}^{-}\right)=q\right) \in \mathcal{J} \times \mathcal{J}, p \neq q$

$$
V_{p}\left(z\left(t_{i}\right)\right) \leq \mu_{p} V_{q}\left(z\left(t_{i}^{-}\right)\right)
$$

then the system (5) is asymptotically stable for any switching signal with MDADT

$$
\tau_{a p}>\tau_{a p}^{*} \triangleq \lim _{w \rightarrow T}\left(-w \frac{\ln \mu_{p}}{\ln \left(1-\lambda_{p} w\right)}\right) .
$$

Proof. This lemma can be proven directly from the definition of the delta operator switched systems in (5), (6), (7), and Lemma 1 and 2.

Remark 4. According to Lemma 1, 2 and 4, the switched systems reaching stable is subject to some conditions even when all the subsystems are stable. Actually, many works have shown that the switched systems may be unstable even when all the subsystems are stable. Similarly, the switched topologies cannot guarantee to reach a consensus even when each topology is with its own proper conditions.

Secondly, we consider the situation (B1) that some subsystems are unstable in the delta operator switched systems. In this situation, the value of $\sigma$ in the system (5) is from the finite set $\mathcal{J}=\{1, \ldots, m\}$, where $m$ is the number of subsystems including $m-r$ unstable subsystems. For convenience, we assume that the $1^{s t}, 2^{\text {nd }}, \ldots, r^{\text {th }}$ subsystem are stable, denoted as $\mathscr{S}$, and the $(r+1)^{t h}, \ldots, m^{\text {th }}$ subsystem are unstable, denoted as $\mathscr{U}$. We have the following theorem for this situation:

Theorem 1. Consider the delta operator switched systems (5), and given constants $\alpha_{p}, \mu_{p}>1, p \in \mathcal{J}$. Suppose that there exist $C^{1}$ functions $V_{\sigma(t)}$, and class $K_{\infty}$ functions $k_{1 p}$ and $k_{2 p}$, such that $\forall p \in \mathcal{J}$,

$$
\begin{gathered}
k_{1 p}(\|z(t)\|) \leq V_{p}(\|z(t)\|) \leq k_{2 p}(\|z(t)\|) \\
\delta V_{p}(z(t)) \leq \alpha_{p} V_{p}(z(t))
\end{gathered}
$$


and $\forall\left(\sigma\left(t_{i}\right)=p, \sigma\left(t_{i}^{-}\right)=q\right) \in \mathcal{J} \times \mathcal{J}, \mathrm{p} \neq \mathrm{q}$

$$
V_{p}\left(z\left(t_{i}\right)\right) \leq \mu_{p} V_{q}\left(z\left(t_{i}^{-}\right)\right)
$$

Then the system is globally uniformly asymptotically stable with marginal $\gamma^{*}$ under the following conditions:

$$
\begin{cases}\tau_{a p}>\lim _{w \rightarrow T}\left(-w \frac{\ln \mu_{p}}{\ln \left(1+\alpha_{p} w\right)}\right) & \left(\alpha_{p} \in(-1 / T, 0) \text { when } T \neq 0, \text { or } \alpha_{p}<0 \text { when } T=0, p \in \mathscr{S}\right) \\ \tau_{a p}>\tau_{a p}^{*} & \left(\alpha_{p}>0, \forall \tau_{a p}^{*}>0, p \in \mathscr{U}\right) \\ \frac{T^{-}}{T^{+}}>\frac{\ln \gamma^{+}-\ln \gamma^{*}}{\ln \gamma^{*}-\ln \gamma^{-}} & \left(0<\gamma^{-}<\gamma^{*}<1\right)\end{cases}
$$

where $T^{-}=\sum_{p=1}^{r} T_{p}(t, 0), T^{+}=\sum_{p=r+1}^{m} T_{p}(t, 0), \bar{\varsigma}_{\sigma\left(t_{i}\right)}=\lim _{w \rightarrow T}\left(1+\alpha_{\sigma\left(t_{i}\right)} w\right)^{1 / w}, \gamma^{-}=\max _{p \in \mathscr{S}}\left(\mu_{p}^{1 / \tau_{a p}} \bar{\varsigma}_{p}\right), \gamma^{+}=\max _{p \in \mathscr{U}}\left(\mu_{p}^{1 / \tau_{a p}} \bar{\varsigma}_{p}\right)$, and $t_{1}, t_{2}, t_{3}, \cdots$ are successive sampling times for the sampled systems, or successive switching time for the continuous systems.

Proof. Suppose $t_{1}, t_{2}, t_{3}, \cdots$ are successive sampling times for the sampled systems, or successive switching time for the continuous systems, then :

$\sigma\left(t_{l}\right)=\sigma\left(t_{l-1}\right)$ stands for no systems switching between the sampling time $t_{l}$ and $t_{l-1}$, and $\mu_{\sigma\left(t_{l}\right)}=1$;

$\sigma\left(t_{l}\right)=p, \sigma\left(t_{l-1}\right)=q, p \neq q$ stands for a systems changing between the time $t_{l}$ and $t_{l-1}$, and this case is denoted as $\sigma\left(t_{l}\right)=p, \sigma\left(t_{l}^{-}\right)=q$, and $\mu_{p}>1$.

According to the condition $\tau_{a p}>\lim _{w \rightarrow T}\left(-w \frac{\ln \mu_{p}}{\ln \left(1+\alpha_{p} w\right)}\right)$ in (23), one has

\section{1 .}

According to the condition $\frac{T^{-}}{T^{+}}>\frac{\ln \gamma^{+}-\ln \gamma^{*}}{\ln \gamma^{*-\ln \gamma^{-}}}$in (23), one has

$$
\gamma^{-T^{-}} \gamma^{+T^{+}}<\gamma^{*(t-0)}
$$

The inequality (21)is equivalent to

$$
\delta V_{p}(z(t))=\lim _{w \rightarrow T}\left(\frac{V_{p}(z(t+w))-V_{p}(z(t))}{w}\right) \leq \alpha_{p} V_{p}(z(t)) .
$$

Then we have

$$
V_{p}(z(t+w)) \leq\left(1+w \alpha_{p}\right) V_{p}(z(t))
$$

For any $t>0$, let $t_{0}=0$. For $\forall t \in\left[t_{l}, t_{l+1}\right)$, let $V_{\sigma\left(t_{l}\right)}\left(t_{l}\right)=V_{\sigma\left(t_{l}\right)}\left(x\left(t_{l}\right)\right)$. Through (22) and (26), we can get

$$
\begin{aligned}
& V_{\sigma(t)}(t) \\
& \leq\left(1+w \alpha_{p}\right)^{\left(t-t_{l}\right) / w} V_{\sigma\left(t_{l}\right)}\left(t_{l}\right) \\
& \leq \bar{\varsigma}_{\sigma\left(t_{l}\right)}^{\left(t-t_{l}\right)} V_{\sigma\left(t_{l}\right)}\left(t_{l}\right) \\
& \leq \mu_{\sigma\left(t_{l}\right)} \bar{S}_{\sigma\left(t_{l}\right)}^{\left(t-t_{l}\right)} V_{\sigma\left(t_{l}\right)}\left(t_{l}^{-}\right) \\
& \leq \mu_{\sigma\left(t_{l}\right)} \bar{S}_{\sigma\left(t_{l}\right)}^{\left(t-t_{l}\right)} \bar{\varsigma}_{\sigma\left(t_{l-1}\right)}^{\left(t_{l}-t_{-1}\right)} V_{\sigma\left(t_{l-1}\right)}\left(t_{l-1}\right) \\
& \leq \mu_{\sigma\left(t_{l}\right)} \mu_{\sigma\left(t_{l-1}\right)} \overline{\boldsymbol{S}}_{\sigma\left(t_{l}\right)}^{\left(t-t_{l}\right)} \overline{\boldsymbol{S}}_{\sigma\left(t_{l-1}\right)}^{\left(t_{l}\right)} V_{\sigma\left(t_{l-1}\right)}\left(t_{l-1}^{-}\right) \\
& \vdots \\
& \leq \prod_{s=1}^{l} \mu_{\sigma\left(t_{s}\right)} \times \overline{\boldsymbol{S}}_{\sigma\left(t_{l}\right)}^{\left(t-t_{l}\right)} \overline{\boldsymbol{S}}_{\sigma\left(t_{l-1}\right)}^{\left(t_{l}-t_{l-1}\right)} \cdots \overline{\boldsymbol{S}}_{\sigma\left(t_{1}\right)}^{\left(t_{2}-t_{1}\right) \bar{\varsigma}_{\sigma(0)}^{\left(t_{1}-0\right)} V_{\sigma(0)}(0) .}
\end{aligned}
$$


According to (4), we can obtain

$$
\begin{aligned}
& V_{\sigma(t)}(t) \\
& \leq \prod_{p=1}^{r} \mu_{p}^{N_{\sigma_{p}}(t, 0)} \prod_{\substack{p=r+1 \\
N}}^{N} \mu_{p}^{N_{\sigma_{p}}(t, 0)} \prod_{p=1}^{r} \bar{\varsigma}_{p}^{T_{p}(t, 0)} \prod_{p=r+1}^{N} \bar{\varsigma}_{p}^{T_{p}(t, 0)} V_{\sigma(0)}(0) \\
& \leq \prod_{p=1}^{r} \mu_{p}^{\left(N_{0 p}+\frac{T_{p}(t, 0)}{\tau_{a p}}\right)} \prod_{p=r+1}^{N} \mu_{p}^{\left(N_{0 p}+\frac{T_{p}(t, 0)}{\tau_{a p}}\right)} \prod_{p=1}^{r} \bar{\varsigma}_{p}^{T_{p}(t, 0)} \prod_{p=r+1}^{N} \bar{\varsigma}_{p}^{T_{p}(t, 0)} V_{\sigma(0)}(0) \\
& \leq \exp \left\{\sum_{p=1}^{N} N_{0 p} \ln \mu_{p}\right\} \prod_{p=1}^{r}\left(\mu_{p}^{\frac{1}{\tau_{a p}}} \bar{\varsigma}_{p}\right)^{T_{p}(t, 0)} \prod_{p=r+1}^{N}\left(\mu_{p}^{\frac{1}{\tau_{a p}}} \bar{\varsigma}_{p}\right)^{T_{p}(t, 0)} V_{\sigma(0)}(0) .
\end{aligned}
$$

Combining (24) and (25) we have

$$
\begin{aligned}
& V_{\sigma(t)}(t) \\
& \leq \exp \left\{\sum_{p=1}^{N} N_{0 p} \ln \mu_{p}\right\} \gamma^{-T^{-}} \gamma^{+T^{+}} V_{\sigma(0)}(0) \\
& \leq \exp \left\{\sum_{p=1}^{N} N_{0 p} \ln \mu_{p}\right\} \gamma^{*\left(t-t_{0}\right)} V_{\sigma(0)}(0) \\
& \leq K \gamma^{*\left(t-t_{0}\right)} V_{\sigma(0)}(0)
\end{aligned}
$$

where $K=\exp \left\{\sum_{p=1}^{N} N_{0 p} \ln \mu_{p}\right\}$. We can then conclude that $V_{\sigma(t)}(t)$ converges to zero with marginal $\gamma^{*}$ as $t \rightarrow \infty$. The proof is completed.

\subsubsection{Problem conversion}

In order to convert the consensus problems to the stability problems in delta operator switched systems, we first convert the consensus problems into stability problems and then rewrite them in the form of delta operator switched systems.

Theorem 2. For the directed network $G(t)$, if the system

$$
\dot{z}(t)=\tilde{H}(t) z(t), z\left(t_{0}\right)=z(0) .
$$

is globally uniformly asymptotically stable, where

$$
\begin{aligned}
& \tilde{H}(t)=\left[\begin{array}{ll}
0 & I \\
-\beta_{0} H & \hat{I}
\end{array}\right](t), \\
& \hat{I}=\left[\begin{array}{llll}
-\beta_{1} I & -\beta_{2} I & \cdots & -\beta_{G-1} I
\end{array}\right],
\end{aligned}
$$

and $z(t)$ is the state vector that contains the state differences of agents, then the consensus of the multi-agent systems (1) is reached under the consensus algorithm (2) for all initial conditions.

Proof. For $\forall k \in \mathcal{K}, \forall i \in\{1, \ldots, n-1\}$, let

$$
\left\{\begin{array}{l}
z_{i}^{(k)}(t)=x_{i}^{(k)}(t)-x_{n}^{(k)}(t), \\
z^{(k)}(t)=\left[z_{1}^{(k)}(t)^{T}, \ldots, z_{n-1}^{(k)}(t)^{T}\right]^{T}, \\
z(t)=\left[z^{(0)}(t)^{T}, \ldots, z^{(G-1)}(t)^{T}\right]^{T},
\end{array}\right.
$$


we have

$$
\begin{aligned}
& \dot{z}^{(k)}(t) \\
= & {\left[\dot{z}_{1}^{(k)}(t)^{T}, \ldots, \dot{z}_{n-1}^{(k)}(t)^{T}\right]^{T} } \\
= & {\left[z_{1}^{(k+1)}(t)^{T}, \ldots, z_{n-1}^{(k+1)}(t)^{T}\right]^{T} } \\
= & z^{(k+1)}(t), \text { when } k<G-1 .
\end{aligned}
$$

because of

$$
\begin{aligned}
& \dot{z}_{i}^{(k)}(t) \\
= & \dot{x}_{i}^{(k)}(t)-\dot{x}_{n}^{(k)}(t) \\
= & x_{i}^{(k+1)}(t)-x_{n}^{(k+1)}(t) \\
= & z_{i}^{(k+1)}(t), \text { when } k<G-1 .
\end{aligned}
$$

Rewrite the $i^{\text {th }}$ row vector of $L$ and $H$ as

$$
\left\{\begin{array}{l}
L_{i}(t)=\left[l_{i 1}(t), l_{i 2}(t), \ldots, l_{i n}(t)\right], \\
H_{i}(t)=\left[h_{i 1}(t), h_{i 2}(t), \ldots, h_{i n-1}(t)\right],
\end{array}\right.
$$

then, we can get

$$
\begin{aligned}
& \dot{z}^{(G-1)}(t) \\
= & {\left[\dot{z}_{1}^{(G-1)}(t)^{T}, \ldots, \dot{z}_{n-1}^{(G-1)}(t)^{T}\right]^{T} } \\
= & -\beta_{0} H(t) z^{(0)}(t)+\hat{I}\left[z^{(1)}(t)^{T}, \ldots, z^{(G-1)}(t)^{T}\right]^{T},
\end{aligned}
$$

according to

$$
\begin{aligned}
& \dot{z}_{i}^{(G-1)}(t) \\
= & \dot{x}_{i}^{(G-1)}(t)-\dot{x}_{n}^{(G-1)}(t) \\
= & \beta_{0} \sum_{j \in \mathcal{N}_{i}(t)} a_{i j}\left(x_{j}^{(0)}(t)-x_{i}^{(0)}(t)\right)-\beta_{1} x_{i}^{(1)}(t)-\cdots-\beta_{G-1} x_{i}^{(G-1)}(t) \\
& \left.-\beta_{0} \sum_{j \in \mathcal{N}_{n}(t)} a_{n j}\left(x_{j}^{(0)}(t)-x_{n}^{(0)}(t)\right)-\beta_{1} x_{n}^{(1)}(t)-\cdots-\beta_{G-1} x_{n}^{(G-1)}(t)\right) \\
= & -\beta_{0}\left(L_{i}(t)-L_{n}(t)\right) x^{(0)}(t)-\beta_{1} z_{i}^{(1)}(t)-\cdots-\beta_{G-1} z_{i}^{(G-1)}(t) \\
= & -\beta_{0} H_{i}(t) z_{i}^{(0)}(t)-\beta_{1} z_{i}^{(1)}(t)-\cdots-\beta_{G-1} z_{i}^{(G-1)}(t) .
\end{aligned}
$$

From (33) and (34), (30) can be established. If the system in (30) is globally uniformly asymptotically stable, the consensus of the multi-agent systems (1) is reached under the consensus algorithm (2) for all initial conditions by Definition 1. This completes the proof.

For a fixed topology, the network $\mathcal{G}$ and systems (30)(31) are time-invariant. In this situation, reaching a consensus under all initial conditions requires adequate coefficients $\beta_{1}, \cdots, \beta_{G-1}$ to make the system (30) be globally uniformly asymptotically stable. In this paper, we call such coefficients $\beta_{1}, \cdots, \beta_{G-1}$ as proper coefficients when they make 
the system stable successfully; otherwise, we call them improper coefficients. For the switching topologies, the coefficients $\beta_{1}, \cdots, \beta_{G-1}$ are accordingly called as proper (or improper) coefficients relative to the corresponding topologies in the set $\mathcal{J}$.

From Theorem 2, we can see that, if the system (30) is asymptotically stable, then the multi-agent systems (1) will reach consensus, under all initial conditions when the consensus algorithm in (2) is applied. Systems (30) can be rewritten as

$$
\dot{z}(t)=\hat{A} z(t)+\hat{B}(t) z(t), z\left(t_{0}\right)=z(0) .
$$

where

$$
\begin{gathered}
\hat{A}=\left[\begin{array}{ll}
0 & I \\
0 & 0
\end{array}\right], \\
\hat{B}=\left[\begin{array}{ccccc}
0 & 0 & 0 & \cdots & 0 \\
-\beta_{0} H & -\beta_{1} I & -\beta_{2} I & \ldots & -\beta_{G-1} I
\end{array}\right] .
\end{gathered}
$$

For the sampled data with sample period $T$, the next state after the $k^{\text {th }}$ sampled time is

$$
z\left(t_{k+1}\right)=e^{\hat{A} T} z\left(t_{k}\right)+\int_{0}^{T} e^{A(T-\tau)} \hat{B}\left(t_{k}\right) d \tau z\left(t_{k}\right) .
$$

In order to process the multi-agent systems with a delta method, one can rewrite systems (38) in the following form

where the matrices $A$ and $B$ are defined as

$$
\begin{aligned}
& =\frac{z\left(t_{k+1}\right)-z\left(t_{k}\right)}{T} \\
& =\frac{e^{\hat{A} T} z\left(t_{k}\right)+\int_{0}^{T} e^{\hat{A}(T-\tau)} \hat{B}\left(t_{k}\right) d \tau z\left(t_{k}\right)-z\left(t_{k}\right)}{T} \\
& =\frac{e^{\hat{A} T} z\left(t_{k}\right)-z\left(t_{k}\right)}{T}+\frac{\int_{0}^{T} e^{\hat{A}(T-\tau)} \hat{B}\left(t_{k}\right) d \tau z\left(t_{k}\right)}{T} \\
& =\frac{e^{\hat{A} T}-I}{T} z\left(t_{k}\right)+\frac{\int_{0}^{T} e^{\hat{A}(T-\tau)} \hat{B}\left(t_{k-1}\right) d \tau}{T} z\left(t_{k}\right) \\
& \triangleq A z\left(t_{k}\right)+B_{\sigma} z\left(t_{k}\right),
\end{aligned}
$$

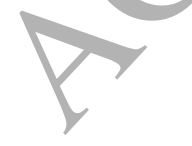

$$
\begin{gathered}
A \triangleq \frac{e^{\hat{A} T}-I}{T}, T \neq 0, \\
B_{\sigma} \triangleq \frac{\int_{0}^{T} e^{\hat{A}(T-\tau)} \hat{B}\left(t_{k}\right) d \tau}{T}, T \neq 0 .
\end{gathered}
$$

According to (39) and the definition of the delta operator, the sampled systems of (35) can be rewritten as

$$
\delta z(t)=A z(t)+B_{\sigma} z(t),
$$


when $T \neq 0$.

Remark 5. If we redefine matrices $A$ and $B$ as $A \triangleq \lim _{w \rightarrow T} \frac{e^{\hat{A} w}-I}{w}$ and $B_{\sigma} \triangleq \lim _{w \rightarrow T} \frac{\int_{0}^{w} e^{\hat{A}(w-\tau)} \hat{B}\left(t_{k}\right) d \tau}{w}$, then the delta systems (40) also represents the continuous-time systems (35), because $A=\lim _{w \rightarrow T} \frac{e^{A w}-I}{w}=\hat{A}, B_{\sigma}=\lim _{w \rightarrow T} \frac{\int_{0}^{w} e^{\hat{A}(w-\tau)} \hat{B}\left(t_{k}\right) d \tau}{w}=\hat{B}(t)$, when $T=0$.

\subsubsection{Solutions to the consensus problems}

Based on the preceding results, we can now present solutions to the original consensus problems. We first consider the situation (A) that all the topologies are with proper coefficients, and then consider the situation (B) that some topologies are with mismatches.

In the first situation, the value of $\sigma$ in the system (40) is from the finite set $\mathcal{J}=\{1, \ldots, m\}$, while $m$ is the number of topologies exclusive of those with mismatches. This corresponds to the situation (A1) in switched systems.

Theorem 3. For given constants $\lambda_{p} \in(0,1 / T)$ when $T \neq 0$, or $\lambda_{p}>0$ when $T=0, \mu_{p}>1$, and the switched topologies $\mathcal{G}_{p}$ of the multi-agent systems, $p \in \mathcal{J}$, if there exist symmetric matrices $P_{p}>0, \forall p \in \mathcal{J}$, such that

$$
\left(P_{p}\left(A+B_{p}\right)+\left(A+B_{p}\right)^{T} P_{p}+T\left(A+B_{p}\right)^{T} P_{p}\left(A+B_{p}\right)\right) \leq-\lambda_{p} P_{p}
$$

and $\forall\left(\sigma\left(t_{i}\right)=p, \sigma\left(t_{i}^{-}\right)=q\right) \in \mathcal{J} \times \mathcal{J}, p \neq q$, such that

$$
P_{p} \leq \mu_{p} P_{q}
$$

then the multi-agent systems (1) under consensus algorithm (2) will reach consensus when

$$
\tau_{a p}>\mathcal{T}_{a p} \triangleq \lim _{w \rightarrow T}\left(-w \frac{\ln \mu_{p}}{\ln \left(1-\lambda_{p} w\right)}\right)
$$

Proof. A multiple Lyapunov function is constructed as

$$
V_{p}(z(t))=z^{T}(t) P_{p} z(t)
$$

From the definition of the delta operator (6), one can get

1) When $T \neq 0$.

$$
\delta V_{p}=\left\{\begin{array}{cc}
\dot{V}_{p}, & T=0 . \\
\frac{V_{p}(z(t+T))-V_{p}(z(t))}{T}, & T \neq 0 .
\end{array}\right.
$$

According to (38) and (39), we can get

$$
\begin{aligned}
& z(t+T) \\
= & z(t)+T\left(A+B_{p}\right) z(t) \\
= & \left(I+T\left(A+B_{p}\right)\right) z(t) .
\end{aligned}
$$


According to (44) and (46), we have

$$
\begin{aligned}
& V_{p}(z(t+T))-V_{p}(z(t)) \\
= & z(t+T)^{T} P_{p} z(t+T)-z(t)^{T} P_{p} z(t) \\
= & \left(\left(I+T\left(A+B_{p}\right)\right) z(t)\right)^{T} P_{p}\left(I+T\left(A+B_{p}\right)\right) z(t)-z(t)^{T} P_{p} z(t) \\
= & z(t)^{T} P_{p} z(t)+z(t)^{T} P_{p}\left(T\left(A+B_{p}\right)\right) z(t)+z(t)^{T}\left(T\left(A+B_{p}\right)\right)^{T} P_{p} z(t) \\
& +z(t)^{T}\left(T\left(A+B_{p}\right)\right)^{T} P_{p}\left(T\left(A+B_{p}\right)\right) z(t)-z(t)^{T} P_{p} z(t) \\
= & z(t)^{T} P_{p}\left(T\left(A+B_{p}\right)\right) z(t)+z(t)^{T}\left(T\left(A+B_{p}\right)\right)^{T} P_{p} z(t) \\
& +z(t)^{T}\left(T\left(A+B_{p}\right)\right)^{T} P_{p}\left(T\left(A+B_{p}\right)\right) z(t) \\
= & z(t)^{T}\left(P_{p}\left(T\left(A+B_{p}\right)\right)+\left(T\left(A+B_{p}\right)\right)^{T} P_{p}+\left(T\left(A+B_{p}\right)\right)^{T} P_{p}\left(T\left(A+B_{p}\right)\right)\right) z(t)
\end{aligned}
$$

Combing (45) and (47), we can get

$$
\delta V_{p}(z(t))=z(t)^{T}\left(P_{p}\left(A+B_{p}\right)+\left(A+B_{p}\right)^{T} P_{p}+\left(A+B_{p}\right)^{T} P_{p} T\left(A+B_{p}\right)\right) z(t) .
$$

From (41) and (48), we have

$$
\delta V_{p}(z(t)) \leq-\lambda_{p} V_{p}(z(t))
$$

2) When $T=0$.

From (41) and (44), we can get

$$
\begin{aligned}
& \dot{V}_{p}(z(t)) \\
= & \dot{z}^{T}(t) P_{p} z(t)+z^{T}(t) P_{p} \dot{z}(t) \\
= & z^{T}(t) \tilde{H}_{p}^{T} P_{p} z(t)+z^{T}(t) P_{p} \tilde{H}_{p} z(t) \\
= & z^{T}(t)\left(\tilde{H}_{p}^{T} P_{p}+P_{p} \tilde{H}_{p}\right) z(t) \\
\leq & -\lambda_{p} z^{T}(t) P_{p} z(t) \\
\leq & -\lambda_{p} V_{p}(z(t)) .
\end{aligned}
$$

I.e.

$$
\delta V_{p}(z(t)) \leq-\lambda_{p} V_{p}(z(t))
$$

Combining the results for $T \neq 0$ and $T=0$, we can obtain

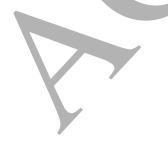

$$
\delta V_{p}(z(t)) \leq-\lambda_{p} V_{p}(z(t))
$$

Based on (42) and (44), one can get

$$
V_{p}\left(z\left(t_{i}\right)\right) \leq \mu_{p} V_{q}\left(z\left(t_{i}\right)\right)
$$

and 


$$
\lambda_{\min }\left(P_{p}\right)\|z(t)\|^{2} \leq V_{p}(z(t)) \leq \lambda_{\max }\left(P_{p}\right)\|z(t)\|^{2} .
$$

Combining (50), (51), (52), and Lemma 4, we can establish Theorem 3.

Using a similar process, we can establish and prove Corollary 1 below when using a common $P$ to replace $P_{p}$.

Corollary 1. For the switched topologies $\mathcal{G}_{p}$ of the multi-agent systems, $p \in \mathcal{J}$, if there exist a symmetric matrix $P>0$, such that

$$
\left(P\left(A+B_{p}\right)+\left(A+B_{p}\right)^{T} P+T\left(A+B_{p}\right)^{T} P\left(A+B_{p}\right)\right)<0,
$$

then the multi-agent systems (1) under consensus algorithm (2) will reach consensus.

In the second situation, some topologies are with mismatches and the value of $\sigma$ in the system (40) is from the finite set $\mathcal{J}=\{1, \ldots, m\}$, where $m$ is the number of topologies including $m-r$ topologies with mismatches. For convenience, we assume that the $1^{s t}, 2^{\text {nd }}, \ldots, r^{\text {th }}$ topology are with no mismatches, denoted as $\mathscr{S}$, and the $(r+1)^{\text {th }}, \ldots, m^{\text {th }}$ topology are with mismatches, denoted as $\mathscr{U}$. This corresponds to the situation (B1) in switched systems.

In this situation, we have the following Theorem.

Theorem 4. For the switched topologies $\mathcal{G}_{p}$ of the multi-agent systems, and give constants $\alpha_{p}, \mu_{p}>1, p \in \mathcal{J}$. If there exist symmetric matrices $P_{p}>0, \forall p \in \mathcal{J}$, such that

$$
\left(P_{p}\left(A+B_{p}\right)+\left(A+B_{p}\right)^{T} P_{p}+T\left(A+B_{p}\right)^{T} P_{p}\left(A+B_{p}\right)\right) \leq \alpha_{p} P_{p},
$$

and for $\forall\left(\sigma\left(t_{i}\right)=p, \sigma\left(t_{i}^{-}\right)=q\right) \in \mathcal{J} \times \mathcal{T}, \mathbf{p} \neq \mathbf{q}$

$$
P_{p} \leq \mu_{p} P_{q},
$$

then the multi-agent systems (1) under consensus algorithm (2) will reach consensus under conditions:

$$
\begin{cases}\tau_{a p}>\lim _{w \rightarrow T}\left(-w \frac{\ln \mu_{p}}{\ln \left(1+\alpha_{p} w\right)}\right) & \left(\alpha_{p} \in(-1 / T, 0) \text { when } T \neq 0, \text { or } \alpha_{p}<0 \text { when } T=0, p \in \mathscr{S}\right) \\ \tau_{a p}>\tau_{a p}^{*} & \left(\alpha_{p}>0, \forall \tau_{a p}^{*}>0, p \in \mathscr{U}\right) \\ \frac{T}{T^{+}}>\frac{\ln \gamma^{*}-\ln \gamma^{*}}{\ln \gamma^{*}-\ln \gamma^{-}} & \left(0<\gamma^{-}<\gamma^{*}<1\right)\end{cases}
$$

where $T^{-}=\sum_{p=1}^{r} T_{p}(t, 0), T^{+}=\sum_{p=r+1}^{m} T_{p}(t, 0), \overline{\boldsymbol{S}}_{\sigma\left(t_{i}\right)}=\lim _{w \rightarrow T}\left(1+\alpha_{\sigma\left(t_{i}\right)} w\right)^{1 / w}, \gamma^{-}=\max _{p \in \mathscr{S}}\left(\mu_{p}^{1 / \tau_{a p}} \overline{\boldsymbol{S}}_{p}\right), \gamma^{+}=\max _{p \in \mathscr{U}}\left(\mu_{p}^{1 / \tau_{a p}} \overline{\boldsymbol{S}}_{p}\right)$, and $t_{1}, t_{2}, t_{3}, \cdots$ are successive sampling times for the sampled systems, or successive switching time for the continuous systems.

Proof. A multiple Lyapunov function is constructed as

$$
V_{p}(z(t))=z^{T}(t) P_{p} z(t)
$$


Using derivations similar to (47)(48) and (49), we can get

$$
\delta V_{p}(z(t)) \leq \alpha_{p} V_{p}(z(t))
$$

Based on (55) and (57), we obtain

$$
V_{p}\left(z\left(t_{i}\right)\right) \leq \mu_{p} V_{q}\left(z\left(t_{i}\right)\right)
$$

and

$$
\lambda_{\min }\left(P_{p}\right)\|z(t)\|^{2} \leq V_{p}(z(t)) \leq \lambda_{\max }\left(P_{p}\right)\|z(t)\|^{2} .
$$

Combining (58), (59), (60), and Lemma 1, we can establish Theorem 4.

\subsection{Case 2: Systems with a known fault}

In this subsection, we investigate the delta operator method for sýstems with a known fault. Detailed types of faults will be discussed in next subsection. Most of the results in this case can be obtained in a similar way to those in the first case.

Based on (2), we consider the following consensus algorithm

$$
u_{i}(t)=f \beta_{0} \sum_{j \in \mathcal{N}_{i}(t)} a_{i j}(t)\left(x_{j}^{(0)}(t)-x_{i}^{(0)}(t)\right)-f \beta_{1} x_{i}^{(1)}(t)-\cdots-f \beta_{G-1} x_{i}^{(G-1)}(t), i=\mathcal{I} .
$$

When the known positive constant $f \neq 1$, it is implied that the consensus algorithm (2) has a fault. The fault is written in the form of deviation ratio.

Remark 6. Note that for the consensus algorithm (3), we can define the version with the fault as:

$$
u_{i}(t)=f \beta_{0} \sum_{j \in \mathcal{N}_{\mathrm{i}}(\mathrm{t})} a_{i j}(t)\left(x_{j}^{(0)}(t)-x_{i}^{(0)}(t)\right)+\cdots+f \beta_{G-1} \sum_{j \in \mathcal{N}_{\mathrm{i}}(\mathrm{t})} a_{i j}(t)\left(x_{j}^{(G-1)}(t)-x_{i}^{(G-1)}(t)\right) .
$$

Accordingly the systems (35) are replaced by

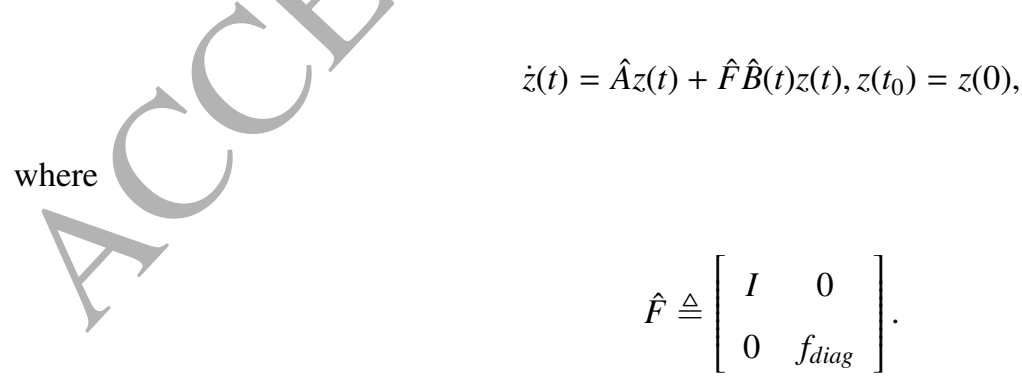

The matrix $f_{\text {diag }}$ is diagonal with elements $f, \ldots, f, f_{\text {diag }}=\operatorname{dig}[f, \cdots, f]$.

Systems (39) and (40) can then be replaced by 


$$
\begin{aligned}
& \frac{z\left(t_{k+1}\right)-z\left(t_{k}\right)}{T} \\
& =\frac{e^{\hat{A} T}-1}{T} z\left(t_{k}\right)+\frac{\hat{F} \int_{0}^{T} e^{\hat{A}(T-\tau)} \hat{B}\left(t_{k-1}\right) d \tau}{T} z\left(t_{k}\right) \\
& =: A z\left(t_{k}\right)+\hat{F} B_{\sigma} z\left(t_{k}\right),
\end{aligned}
$$

and

$$
\delta z(t)=A z(t)+F B_{\sigma} z(t)
$$

respectively, where $F=\hat{F}$.

Using a similar process to establishing Theorem 3 and Corollary 1, we can establish and prove the following Corollary 2 and Corollary 3 for situation (A), when using $F B_{p}$ to replace $B_{p}$.

Corollary 2. For given constants $\lambda_{p} \in(0,1 / T)$ when $T \neq 0$, or $\lambda_{p}>0$ when $T=0, \mu_{p}>1$, and the switched topologies $\mathcal{G}_{p}$ of the multi-agent systems, $p \in \mathcal{J}$, if there exist symmetric matrices $P_{p}>0, \forall p \in \mathcal{J}$, such that

$$
\left(P_{p}\left(A+F B_{p}\right)+\left(A+F B_{p}\right)^{T} P_{p}+T\left(A+F B_{p}\right)^{T} P_{p}\left(A+F B_{p}\right)\right) \leq-\lambda_{p} P_{p},
$$

and $\forall\left(\sigma\left(t_{i}\right)=p, \sigma\left(t_{i}^{-}\right)=q\right) \in \mathcal{J} \times \mathcal{J}, p \neq q$, such that

$$
P_{p} \leq \mu_{p} P_{q}
$$

then the multi-agent systems (1) under consensus algorithm (61), will reach consensus when

$$
>\tau_{a p}^{*} \triangleq \lim _{w \rightarrow T}\left(-w \frac{\ln \mu_{p}}{\ln \left(1-\lambda_{p} w\right)}\right) .
$$

Corollary 3. For the switched topologies $\mathcal{G}_{p}$ of the multi-agent systems, $p \in \mathcal{J}$, if there exist a symmetric matrix $P>0$, such that

$$
\left(P\left(A+F B_{p}\right)+\left(A+F B_{p}\right)^{T} P+T\left(A+F B_{p}\right)^{T} P\left(A+F B_{p}\right)\right)<0,
$$

then the multi-agent systems (1) under consensus algorithm (61) will reach consensus.

Similar to the process of establishing Theorem 4, we can establish and prove the following Corollary 4 for situation (B) when using $F B_{p}$ to replace $B_{p}$.

Corollary 4. For the switched topologies $\mathcal{G}_{p}$ of the multi-agent systems, and give constants $\alpha_{p}, \mu_{p}>1, p \in \mathcal{J}$, if there exist symmetric matrices $P_{p}>0, \forall p \in \mathcal{J}$, such that

$$
\left(P_{p}\left(A+F B_{p}\right)+\left(A+F B_{p}\right)^{T} P_{p}+T\left(A+F B_{p}\right)^{T} P_{p}\left(A+F B_{p}\right)\right) \leq \alpha_{p} P_{p}
$$

and for $\forall\left(\sigma\left(t_{i}\right)=p, \sigma\left(t_{i}^{-}\right)=q\right) \in \mathcal{J} \times \mathcal{J}, \mathrm{p} \neq \mathrm{q}$,

$$
P_{p} \leq \mu_{p} P_{q},
$$


then the multi-agent systems (1) under consensus algorithm (2) will reach consensus under conditions:

$$
\begin{cases}\tau_{a p}>\lim _{w \rightarrow T}\left(-w \frac{\ln \mu_{p}}{\ln \left(1+\alpha_{p} w\right)}\right) & \left(\alpha_{p} \in(-1 / T, 0) \text { when } T \neq 0, \text { or } \alpha_{p}<0 \text { when } T=0, p \in \mathscr{S}\right) \\ \tau_{a p}>\tau_{a p}^{*} & \left(\alpha_{p}>0, \forall \tau_{a p}^{*}>0, p \in \mathscr{U}\right) \\ \frac{T^{-}}{T^{+}}>\frac{\ln \gamma^{+}-\ln \gamma^{*}}{\ln \gamma^{*}-\ln \gamma^{-}} & \left(0<\gamma^{-}<\gamma^{*}<1\right)\end{cases}
$$

where $T^{-}=\sum_{p=1}^{r} T_{p}(t, 0), T^{+}=\sum_{p=r+1}^{m} T_{p}(t, 0), \overline{\boldsymbol{\zeta}}_{\sigma\left(t_{i}\right)}=\lim _{w \rightarrow T}\left(1+\alpha_{\sigma\left(t_{i}\right)} w\right)^{1 / w}, \gamma^{-}=\max _{p \in \mathscr{S}}\left(\mu_{p}^{1 / \tau_{a p}} \overline{\boldsymbol{S}}_{p}\right), \gamma^{+}=\max _{p \in \mathscr{U}}\left(\mu_{p}^{1 / \tau_{a p}} \overline{\boldsymbol{S}}_{p}\right)$, and $t_{1}, t_{2}, t_{3}, \cdots$ are successive sampling times for sampled systems or successive switching time for continuous systems.

Theorem 3 ( Corollary 1, Theorem 4) can be regarded as a special situation of Corollary $2(3,4)$ when the consensus algorithm has no fault. Besides, Corollary 2, 3 and 4 are also applicable to continuous-time multi-agent systems $(T=0)$, discrete-time multi-agent networks $(T=1)$ and sampled systems $(T>0)$ under switched topologies (timevariant $\left.B_{p}(t)\right)$ or fixed topologies (time-invariant $B_{p}$ ).

\subsection{Case 3: Systems with unknown faults}

We investigate systems with unknown faults in this subsection. The faults can originate from various sources. We assume that the range of every fault is known. The consensus problem is studied through analysing the types of faults and the aggregate weighting ranges of these faults, based on the results in the first case. A new model with the deviation ratio is proposed. The range of $f$ is known as:

$$
\begin{gathered}
0<f_{d} \leq f \leq f_{u} . \\
f_{d} \leq 1 \leq f_{u} .
\end{gathered}
$$

The faults may originate from coupling (or strength) coefficient $\beta$, measurement inaccuracies of neighboring states $\left(x_{j}-x_{i}\right)$, linking weighting errors $a_{i j}$ and others. The boundaries $f_{d}$ and $f_{u}$ can stand for not only the range of a fault but also the aggregate weighting ranges of various faults.

We define $F_{u} \triangleq \operatorname{diag}\left(1, \cdots, 1, f_{u}, \cdots f_{u}\right), F_{d} \triangleq \operatorname{diag}\left(1, \cdots, 1, f_{d}, \cdots f_{d}\right), F_{0} \triangleq \frac{1}{2}\left(F_{u}+F_{d}\right), F_{1} \triangleq \frac{1}{2}\left(F_{u}-F_{d}\right)$, then

$$
F=F_{0}+E F_{1},
$$

where $E=\operatorname{diag}(1 \cdots 1, e, \cdots, e)$, and $-1 \leqslant e \leqslant 1$.

Based on (71) and (72), systems (64) can be revised as

$$
\begin{aligned}
& \delta z(t) \\
= & A z(t)+F B_{\sigma} z(t) \\
= & A z(t)+\left(F_{0}+E F_{1}\right) B_{\sigma} z(t) \\
= & A z(t)+F_{0} B_{\sigma} z(t)+E F_{1} B_{\sigma} z(t) \\
= & \bar{A}_{\sigma} z(t),
\end{aligned}
$$


where $\bar{A}_{\sigma}=A+F_{0} B_{\sigma}+E F_{1} B_{\sigma}$.

Now we can get Theorem 5 and Corollary 5 below for the robust unknown fault case. They correspond to Situation (A).

Theorem 5. For given constants $\lambda_{p} \in(0,1 / T)$ when $T \neq 0$, or $\lambda_{p}>0$ when $T=0, \mu_{p}>1$, and the switched topologies $\mathcal{G}_{p}$ of the multi-agent systems, $p \in \mathcal{J}$, if there exist symmetric matrices $P_{p}>0$, positive constants $\varepsilon_{p}$, $\forall p \in \mathcal{J}$, such that

$$
\left[\begin{array}{cccc}
D_{p} & T P_{p}\left(A+F_{0} B_{p}\right)^{T} & \varepsilon_{p} F_{1} & P_{p} B_{p}^{T} \\
T\left(A+F_{0} B_{p}\right) P_{p} & -T P_{p} & \varepsilon_{p} T F_{1} & 0 \\
\varepsilon_{p} F_{1}{ }^{T} & \varepsilon_{p} T F_{1}{ }^{T} & -\varepsilon_{p} I & 0 \\
B_{p} P_{p} & 0 & 0 & -\varepsilon_{p} I
\end{array}\right]<0,
$$

where $D_{p}=\left(A+F_{0} B_{p}\right) P_{p}+P_{p}\left(A+F_{0} B_{p}\right)^{T}+\lambda_{p} P_{p}$, and for $\forall\left(\sigma\left(t_{i}\right)=p, \sigma\left(t_{i}^{-}\right)=q\right) \in \mathcal{J} \times \mathcal{J}, p \neq q$, such that

$$
P_{p}^{-1} \leq \mu_{p} P_{q}^{-1}
$$

then the multi-agent systems (1) under consensus algorithm (61) with fault tolerance (71) will reach consensus when

$$
\tau_{a p}>\tau_{a p}^{*} \triangleq \lim _{w \rightarrow T}\left(-w \frac{\ln \mu_{p}}{\ln \left(1-\lambda_{p} w\right)}\right) .
$$

Proof. According to the Schur Complement lemma, one can get that systems (74) are equivalent to

$$
\begin{aligned}
& \qquad R_{p}-\left[\begin{array}{cc}
\varepsilon_{p} F_{1} & P_{p} B_{p}^{T} \\
\varepsilon_{p} T F_{1} & 0
\end{array}\right]\left[\begin{array}{cc}
-\varepsilon_{p} I & 0 \\
0 & -\varepsilon_{p} I
\end{array}\right]^{-1} S_{p}<0, \\
& \text { where } R_{p}=\left[\begin{array}{cc}
D_{p} & T P_{p}\left(A+F_{0} B_{p}\right)^{T} \\
T\left(A+F_{0} B_{p}\right) P_{p} & -T P_{p}
\end{array}\right], S_{p}=\left[\begin{array}{cc}
\varepsilon_{p} F_{1}{ }^{T} & \varepsilon_{p} T F_{1}{ }^{T} \\
B_{p} P_{p} & 0
\end{array}\right] . \\
& \text { This can be further written as }
\end{aligned}
$$

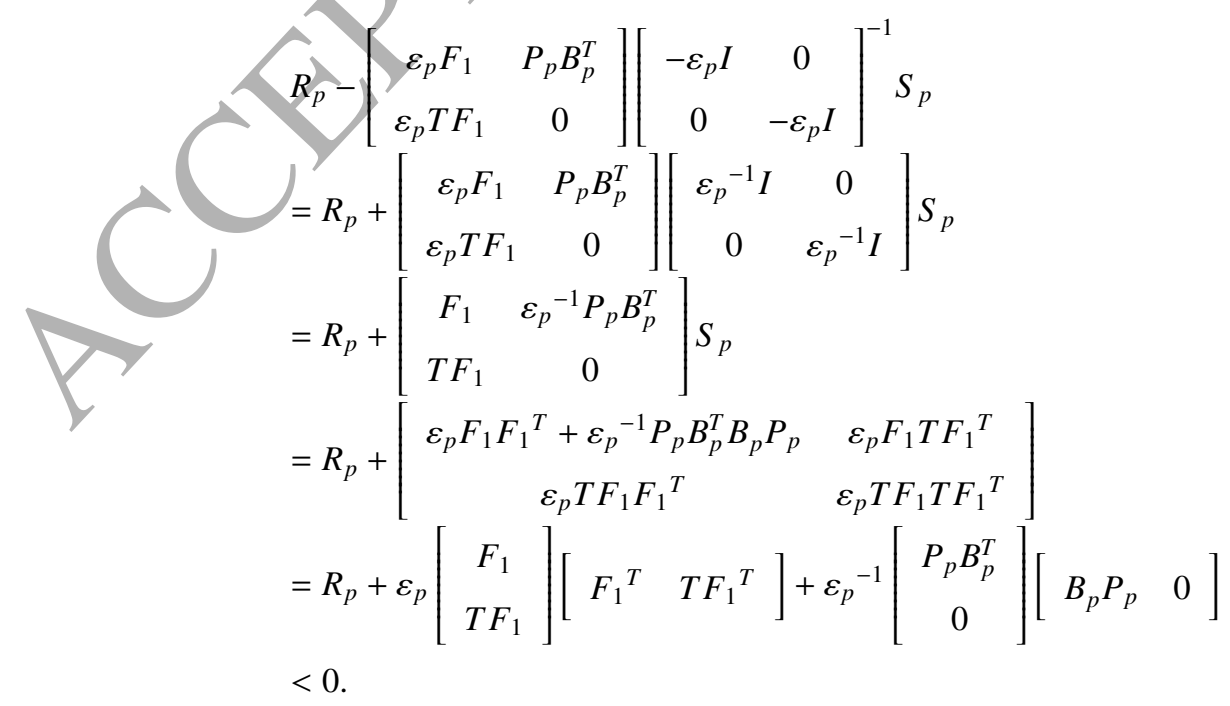


From Lemma 3, one can get

$$
R_{p}+\left[\begin{array}{c}
F_{1} \\
T F_{1}
\end{array}\right] E\left[\begin{array}{ll}
B_{p} P_{p} & 0
\end{array}\right]+\left[\begin{array}{c}
P_{p} B_{p}^{T} \\
0
\end{array}\right] E^{T}\left[\begin{array}{cc}
F_{1}{ }^{T} & T F_{1}{ }^{T}
\end{array}\right]<0
$$

This can also be further represented as

$$
\begin{aligned}
& R_{p}+\left[\begin{array}{cc}
F_{1} E B_{p} P_{p} & 0 \\
T F_{1} E B_{p} P_{p} & 0
\end{array}\right]+\left[\begin{array}{cc}
P_{p} B_{p}^{T} E^{T} F_{1}^{T} & T P_{p} B_{p}^{T} E^{T} F_{1}^{T} \\
0 & 0
\end{array}\right] \\
& =\left[\begin{array}{c}
\hat{D_{p}} \\
T P_{p}\left(A+F_{0} B_{p}+F_{1} E B_{p}\right)^{T} \\
T\left(A+F_{0} B_{p}+F_{1} E B_{p}\right) P_{p}
\end{array}\right] \\
& <0,
\end{aligned}
$$

where $\hat{D_{p}}=\left(A+F_{0} B_{p}+F_{1} E B_{p}\right) P_{p}+P_{p}\left(A+F_{0} B_{p}+F_{1} E B_{p}\right)^{T}+\lambda_{p} P_{p}$.

Using $\operatorname{diag}\left\{P_{p}{ }^{-1}, I\right\}$ to pre- and post-multiply both sides of systems (79), respectively, one has

$$
\begin{aligned}
& {\left[\begin{array}{cc}
\check{D_{p}} & T\left(A+F_{0} B_{p}+F_{1} E B_{p}\right)^{T} \\
T\left(A+F_{0} B_{p}+F_{1} E B_{p}\right) & -T P_{p}
\end{array}\right]} \\
& <0 .
\end{aligned}
$$

where $\check{D_{p}}=P_{p}^{-1}\left(A+F_{0} B_{p}+F_{1} E B_{p}\right)+\left(A+F_{0} B_{p}+F_{1} E B_{p}\right)^{T} P_{p}^{-1}+\lambda_{p} P_{p}^{-1}$.

According to the Schur complement lemma, systems (80) are equivalent to

$$
\begin{aligned}
& P_{p}^{-1}\left(A+F_{0} B_{p}+F_{1} E B_{p}\right)+\left(A+F_{0} B_{p}+F_{1} E B_{p}\right)^{T} P_{p}^{-1}+\lambda_{p} P_{p}^{-1} \\
& +T\left(A+F_{0} B_{p}+F_{1} E B_{p}\right)^{T}\left(T P_{p}\right)^{-1} T\left(A+F_{0} B_{p}+F_{1} E B_{p}\right) \\
& =P_{p}^{-1}\left(A+F_{0} B_{p}+F_{1} E B_{p}\right)+\left(A+F_{0} B_{p}+F_{1} E B_{p}\right)^{T} P_{p}^{-1}+\lambda_{p} P_{p}^{-1} \\
& +T\left(A+F_{0} B_{p}+F_{1} E B_{p}\right)^{T} P_{p}^{-1}\left(A+F_{0} B_{p}+F_{1} E B_{p}\right) \\
& <0 .
\end{aligned}
$$

Denoting $\bar{P}_{p}=P_{p}^{-1}$, one can get $\bar{P}_{p}>0$ and systems (81) are equivalent to

$$
\begin{aligned}
& \bar{P}_{p}\left(A+F_{0} B_{p}+F_{1} E B_{p}\right)+\left(A+F_{0} B_{p}+F_{1} E B_{p}\right)^{T} \bar{P}_{p} \\
& +\lambda_{p} \bar{P}_{p}+T\left(A+F_{0} B_{p}+F_{1} E B_{p}\right)^{T} \bar{P}_{p}\left(A+F_{0} B_{p}+F_{1} E B_{p}\right) \\
& <0 .
\end{aligned}
$$

Based on systems (73) and (82), one has

$$
\bar{P}_{p}\left(A+F B_{p}\right)+\left(A+F B_{p}\right)^{T} \bar{P}_{p}+\lambda_{p} \bar{P}_{p}+T\left(A+F B_{p}\right)^{T} \bar{P}_{p}\left(A+F B_{p}\right)<0 .
$$

i.e.

$$
\bar{P}_{p}\left(A+F B_{p}\right)+\left(A+F B_{p}\right)^{T} \bar{P}_{p}+T\left(A+F B_{p}\right)^{T}\left(A+F B_{p}\right) \bar{A}_{p}<-\lambda_{p} \bar{P}_{p} .
$$


A multiple Lyapunov function is constructed as

$$
V_{p}(z(t))=z(t)^{T} \bar{P}_{p} z(t)
$$

Based on systems (75), one has

$$
\bar{P}_{p} \leq \mu_{p} \bar{P}_{q}
$$

Combining (84), (85) and (86) and Corollary 2, we can establish Theorem 5. The proof is completed.

Using a similar process, we can establish and prove Corollary 5 below when using a common $P$ to replace $P_{p}$.

Corollary 5. For the switched topologies $\mathcal{G}_{p}$ of the multi-agent systems, $p \in \mathcal{J}$, if there exist a symmetric matrix $P>0$, positive constants $\varepsilon_{p}, \forall p \in \mathcal{J}$, such that

$$
\left[\begin{array}{cccc}
\overline{D_{p}} & T P_{p}\left(A+F_{0} B_{p}\right)^{T} & \varepsilon_{p} F_{1} & P B_{p}^{T} \\
T\left(A+F_{0} B_{p}\right) P & -T P & \varepsilon_{p} T F_{1} & 0 \\
\varepsilon_{p} F_{1}^{T} & \varepsilon_{p} T F_{1}^{T} & -\varepsilon_{p} I & 0 \\
B_{p} P & 0 & 0 & -\varepsilon_{p} I
\end{array}\right]<0
$$

where $\overline{D_{p}}=\left(A+F_{0} B_{p}\right) P+P\left(A+F_{0} B_{p}\right)^{T}$, then the multi-agent systems (1) under consensus algorithm (61) with fault tolerance (71) will reach consensus.

Similarly, we can establish and prove the following Corollary 6 for the robust unknown fault case, that corresponds to situation $(\mathrm{B})$.

Corollary 6. For the switched topologies $\mathcal{G}_{p}$ of the multi-agent systems, and the given constants $\alpha_{p}, \mu_{p}>1, p \in \mathcal{J}$, if there exist symmetric matrices $P_{p}>0$, positive constants $\varepsilon_{p}, \forall p \in \mathcal{J}$, such that

$$
\left[\begin{array}{cccc}
D_{p} & T P_{p}\left(A+F_{0} B_{p}\right)^{T} & \varepsilon_{p} F_{1} & P_{p} B_{p}^{T} \\
T\left(A+F_{0} B_{p}\right) P_{p} & -T P_{p} & \varepsilon_{p} T F_{1} & 0 \\
\varepsilon_{p} F_{1}{ }^{T} & \varepsilon_{p} T F_{1}{ }^{T} & -\varepsilon_{p} I & 0 \\
B_{p} P_{p} & 0 & 0 & -\varepsilon_{p} I
\end{array}\right]<0,
$$

where $D_{p}=\left(A+F_{0} B_{p}\right) P_{p}+P_{p}\left(A+F_{0} B_{p}\right)^{T}-\alpha_{p} P_{p}$,

and for $\forall\left(\sigma\left(t_{i}\right)=p, \sigma\left(t_{i}^{-}\right)=q\right) \in \mathcal{J} \times \mathcal{J}, p \neq q$, such that

$$
P_{p}^{-1} \leq \mu_{p} P_{q}^{-1}
$$

then the multi-agent systems (1) under consensus algorithm (61) with fault tolerance (71) will reach consensus with marginal $\gamma^{*}$ under the following conditions:

$$
\begin{cases}\tau_{a p}>\lim _{w \rightarrow T}\left(-w \frac{\ln \mu_{p}}{\ln \left(1+\alpha_{p} w\right)}\right) & \left(\alpha_{p} \in(-1 / T, 0) \text { when } T \neq 0, \text { or } \alpha_{p}<0 \text { when } T=0, p \in \mathscr{S}\right) \\ \tau_{a p}>\tau_{a p}^{*} & \left(\alpha_{p}>0, \forall \tau_{a p}^{*}>0, p \in \mathscr{U}\right) \\ \frac{T^{-}}{T^{+}}>\frac{\ln \gamma^{+}-\ln \gamma^{*}}{\ln \gamma^{*-l n} \gamma^{-}} & \left(0<\gamma^{-}<\gamma^{*}<1\right)\end{cases}
$$


where $T^{-}=\sum_{p=1}^{r} T_{p}(t, 0), T^{+}=\sum_{p=r+1}^{m} T_{p}(t, 0), \overline{\boldsymbol{S}}_{\sigma\left(t_{i}\right)}=\lim _{w \rightarrow T}\left(1+\alpha_{\sigma\left(t_{i}\right)} w\right)^{1 / w}, \gamma^{-}=\max _{p \in \mathscr{S}}\left(\mu_{p}^{1 / \tau_{a p}} \overline{\boldsymbol{S}}_{p}\right), \gamma^{+}=\max _{p \in \mathscr{U}}\left(\mu_{p}^{1 / \tau_{a p}} \overline{\boldsymbol{S}}_{p}\right)$,and $t_{1}, t_{2}, t_{3}, \cdots$ are successive sampling times for the sampled systems or successive switching time for the continuous systems.

Remark 7. If the systems (1) and consensus algorithms (2) and (3) satisfy a Lipschitz condition, these approaches can be applied to the nonlinear multi-agent systems with the aid of matrix inequations [35] or Takagi-Sugeno fuzzy models [39-41].

Remark 8. The existence of $P, P_{p}$ and $\varepsilon_{p}$ is the sufficient condition for reaching consensus. Our results provide reliable conditions that guarantee consensus, especially when the faults are unknown. The linear matrix inequality (LMI) toolbox in MATLAB can be typically used to find proper values for $P, P_{p}$ and $\varepsilon_{p}$

Data transmission and reducing data-transmission in the complex networks and the multi-agent systems are introduced in [10, 42-47]. The network of multi-agent systems can been regarded as an example of the general complex network sometimes. The methods proposed in this paper can also be extended to some general complex networks.

\section{Simulation Results}

In this section, we present two numerical examples in two different situations to demonstrate the effectiveness of the theoretical results. Since the first case and the second case can be seen as the special circumstances of the third case, we only verify the results for the third case, particularly Theorem 5 and Corollary 6, as Corollary 5 is a special circumstance of Theorem 5 .

We adopt the consensus algorithm with full coupling state information and consider the second-order multi-agent systems with four agents. We set $T=0.01$ and $\beta_{0}=1$. In each example, three signal values (low, middle and high) of switching signal $\sigma(t)$ are used to represent three topologies.

Example 1. Situation (A): All the topologies are with unknown faults, proper coefficients and spanning trees.

In this example, the adjacency matrices of the switched topologies are

$$
\text { ( }\left[\begin{array}{llll}
0 & 0 & 0 & 0 \\
1 & 0 & 0 & 0 \\
0 & 1 & 0 & 0 \\
1 & 0 & 0 & 0
\end{array}\right],\left[\begin{array}{cccc}
0 & 0 & 1 & 0 \\
1 & 0 & 0 & 0 \\
0 & 1 & 0 & 0 \\
1 & 0 & 0 & 0
\end{array}\right] \text {, and, }\left[\begin{array}{cccc}
0 & 0 & 1 & 0 \\
1 & 0 & 0 & 0 \\
0 & 1 & 0 & 1 \\
1 & 0 & 0 & 0
\end{array}\right] \text {. }
$$

For this example, the aggregation bias range of all unknown faults is $0.9000 \leq f \leq 1.1000$. When $\beta_{1}=1.2000$, all topologies are with proper coefficients and a spanning tree according to the work [17]. They can reach consensuses separately.

The LMI toolbox in Matlab is used to find different symmetric matrices $P_{p}$ and positive constants $\varepsilon_{p}$ satisfying conditions (74) and (75), for given constant values $\lambda_{1}=0.0100, \lambda_{2}=0.3516$, and $\lambda_{3}=0.4300$, and $\mu_{1}=1.0002$, $\mu_{2}=1.3923, \mu_{3}=1.7750$. The numerical results are shown in Fig. 1. The consensus is reached finally. 


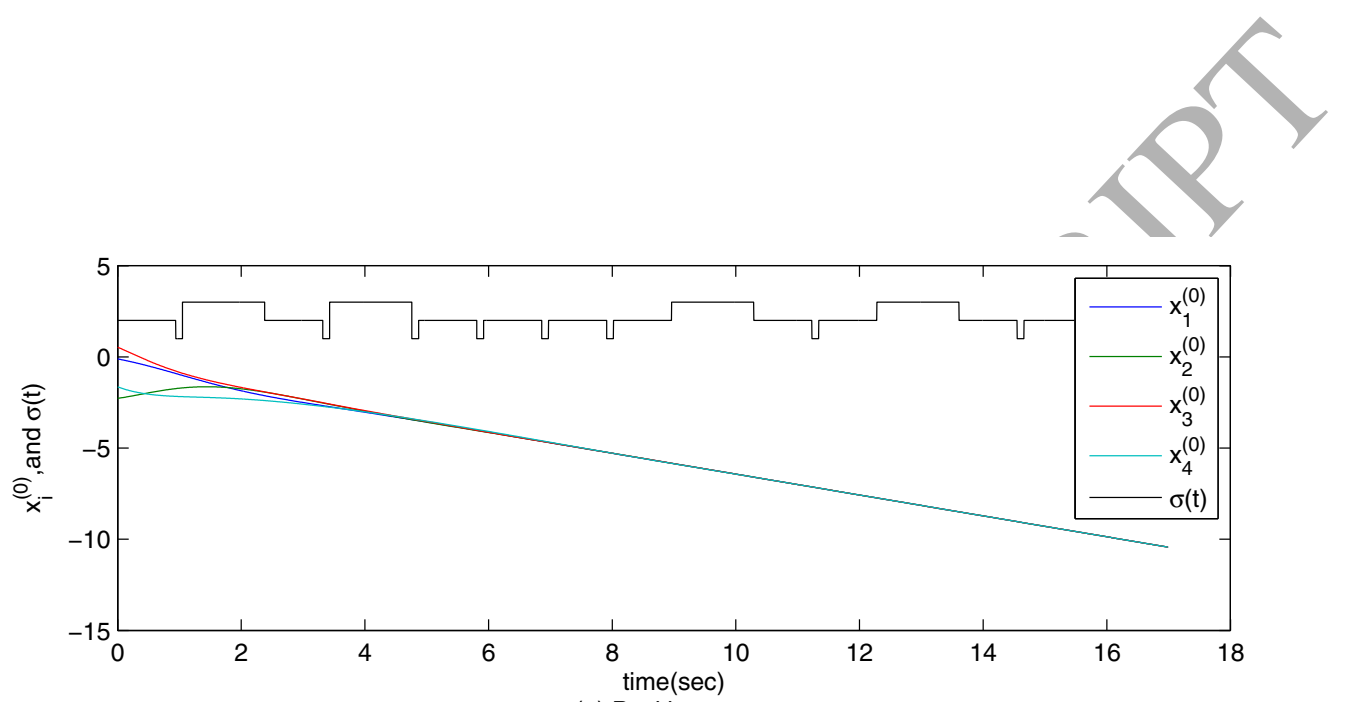

(a) Position states

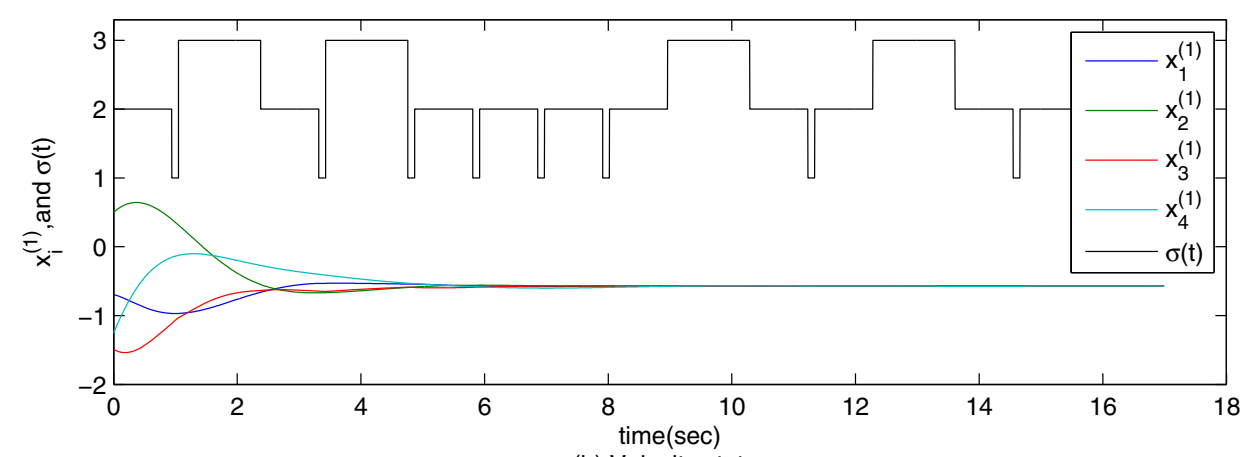

(b) Velocity states

Figure 1: States of agents and switching signal when the topologies are with unknown faults, proper coefficients and spanning trees.

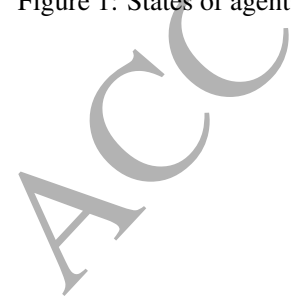


Example 2. Situation (B): All the topologies are with unknown faults. Some of them are with improper coefficients and no spanning tree.

In this example, the adjacency matrices of the switched topologies are

$$
\left[\begin{array}{cccc}
0 & 0 & 0 & 0 \\
1 & 0 & 0 & 0 \\
0 & 1 & 0 & 0 \\
1 & 0 & 0 & 0
\end{array}\right],\left[\begin{array}{cccc}
0 & 0 & 1 & 0 \\
1 & 0 & 0 & 0 \\
0 & 1 & 0 & 0 \\
1 & 0 & 0 & 0
\end{array}\right] \text {, and }\left[\begin{array}{cccc}
0 & 0 & 0 & 0 \\
1 & 0 & 0 & 0 \\
0 & 0 & 0 & 0 \\
1 & 0 & 0 & 0
\end{array}\right]
$$

For this example, the aggregation bias range of all unknown faults is $0.9900 \leq f \leq 1.0100$. When $\beta_{1}=0.3700$. The second topology is with improper coefficients and the third topology has no spanning tree. They cannot reach consensuses separately according to the work [17].

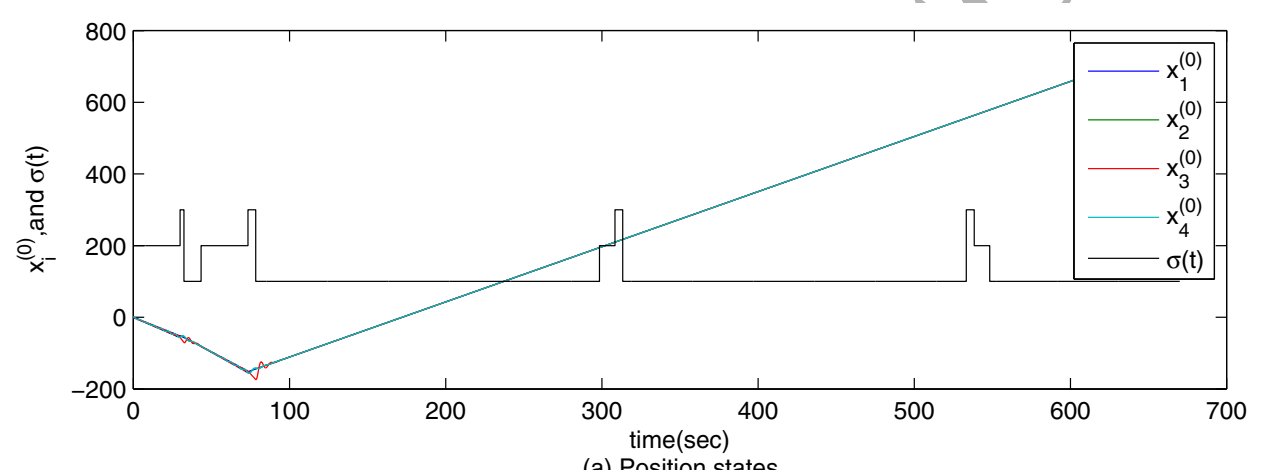

(a) Position states

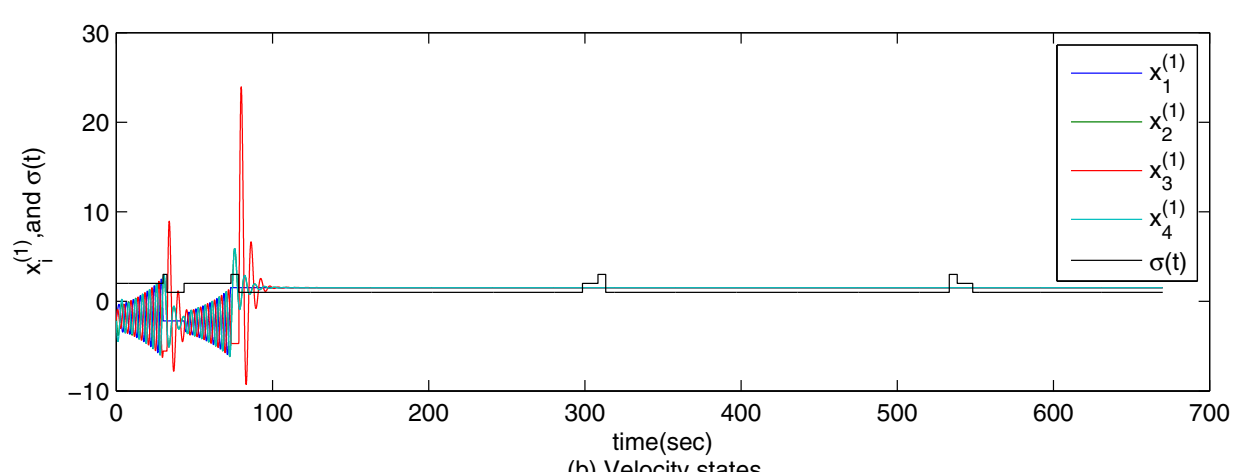

Figure 2: States of agents and switching signal when all the topologies are with unknown faults and some of them are with improper coefficients and no spanning tree.

The LMI toolbox is used to find different symmetric matrices $P_{p}$ and positive constants $\varepsilon_{p}$ satisfying conditions (75) (88) and (89), for given constant values $\alpha_{1}=-0.06500, \alpha_{2}=2.000$, and $\alpha_{3}=1.159$, and $\mu_{1}=1.000, \mu_{2}=2.343$, $\mu_{3}=1.874$. According to the condition in (89), $\frac{T^{-}}{T^{+}}>19.65$. The numerical results in this situation are shown in Fig. 2. In the first 100 seconds, the states of agents tend to diverge, as the dwell time of the agents is far from meeting the conditions in (89). With the running time increasing, the states of agents turn to the consensus when the dwell time of 
the agents is close to the conditions in (89). The consensus is reached finally.

When the number of agents increases, more computing resources and time will be needed to handle these LMIs. Such requirements can be relaxed by, e.g., using fitful hardware and software platforms at different scales. Offloading the computation to off-line is also an efficient method.This is because the feasibility does not depend on the evolution of multi-agents if we can know the ranges of switching topologies in advance.

\section{Conclusion}

In this paper, we study and solve a series of consensus problems for continuous, discrete, or sampled multi-agent systems with faults and mismatches under switched topologies. Using the delta operator method, the consensus problems are unified under a single framework. We demonstrate that consensus can be reached under switched topologies with mismatches and unknown faults, even when some of the topologies have no spanning tree. The approaches proposed in this paper can be extended to deal with other cooperating problems, such as nonlinear multiagent systems.

\section{Acknowledgements}

This work is supported by the National Natural Science Foundation of China ( Grant No.61374117)

\section{References}

[1] P. Delellis, G. Polverino, G. Ustuner, N. Abaid, S. Macr, E. M. Bollt, M. Porfiri, Collective behaviour across animal species, Sci. Rep. 4 (1) (2014) 3723.

[2] J. Zhan, X. Li, Consensus of sampled-data multi-agent networking systems via model predictive control, Automatica 49 (8) (2013) 25022507.

[3] X. Wang, Z. Zeng, Y. Cong, Multi-agent distributed coordination control: Developments and directions via graph viewpoint, Neurocomputing 199 (2016) 204-218.

[4] J. Terán, J. Aguilar, M. Cerrada, Integration in industrial automation based on multi-agent systems using cultural algorithms for optimizing the coordination mechanisms, Comput. Ind. 91 (2017) 11-23.

[5] D. Pardo, C. Angulo, S. del Moral, A. Català, Emerging motor behaviors: Learning joint coordination in articulated mobile robots, Neurocomputing 72 (16) (2009) 3624-3630.

[6] W. Ren, Y. Cao, Distributed coordination of multi-agent networks: emergent problems, models, and issues, Springer Science and Business Media, 2010

[7] D. Zheng, H. Zhang, Q. Zheng, Consensus analysis of multi-agent systems under switching topologies by a topology-dependent average dwell time approach, IET Control Theory Appl. 11 (3) (2017) 429-438.

[8] W. Yu, W. X. Zheng, G. Chen, W. Ren, J. Cao, Second-order consensus in multi-agent dynamical systems with sampled position data, Automatica 47 (7) (2011) 1496-1503.

[9] N. Huang, Z. Duan, G. Chen, Some necessary and sufficient conditions for consensus of second-order multi-agent systems with sampled position data, Automatica 63 (2016) 148-155.

[10] Z. Lei, Z. Wang, H. Gao, F. E. Alsaadi, Finite-horizon $h_{\infty}$ consensus control of time-varying multiagent systems with stochastic communication protocol, IEEE Trans. Cybern. 47 (8) (2017) 1830-1840. 
[11] R. Olfati-Saber, R. M. Murray, Consensus problems in networks of agents with switching topology and time-delays, IEEE Trans. Autom. Control 49 (9) (2004) 1520-1533.

[12] W. Ren, R. W. Beard, Consensus seeking in multiagent systems under dynamically changing interaction topologies, IEEE Trans. Autom. Control 50 (5) (2005) 655-661.

[13] W. Ren, E. Atkins, Second-order consensus protocols in multiple vehicle systems with local interactions, in: AIAA Guid.,Navigation Control Conf. Exhibit., 2005, pp. 15-18.

[14] D. Zheng, H. Zhang, Research on the transformation of control protocols among three kinds of cooperative control for multi-agent systems, in: 2016 8th International Conference on Intelligent Human-Machine Systems and Cybernetics (IHMSC), IEEE, 2016, pp. 301-304.

[15] W. Ren, K. L. Moore, Y. Chen, High-order and model reference consensus algorithms in cooperative control of multivehicle systems, ASME J.f Dyn. Syst., Meas., Control 129 (5) (2007) 678-688.

[16] Y. Wu, J. Hu, Y. Zhang, Y. Zeng, Interventional consensus for high-order multi-agent systems with unknown disturbances on coopetition networks, Neurocomputing 194 (2016) 126-134.

[17] W. Yu, G. Chen, M. Cao, Brief paper: Some necessary and sufficient conditions for second-order consensus in multi-agent dynamical systems, Automatica 46 (6) (2010) 1089-1095.

[18] Y. Cao, Y. Sun, Consensus of discrete-time third-order multi-agent systems in directed networks, Neurocomputing 177 (2016) $394-400$.

[19] Q. Shen, B. Jiang, P. Shi, J. Zhao, Cooperative adaptive fuzzy tracking control for networked unknown nonlinear multiagent systems with time-varying actuator faults, IEEE Trans. Fuzzy Syst. 22 (3) (2014) 494-504.

[20] I. Saboori, K. Khorasani, Actuator fault accommodation strategy for a team of multi-agent systems subject to switching topology, Automatica 62 (C) (2015) 200-207.

[21] S. Chen, D. W. Ho, L. Li, M. Liu, Fault-tolerant consensus of multi-agent system with distributed adaptive protocol, IEEE Trans. cybernetics 45 (10) (2015) 2142-2155.

[22] X. Wang, G.-H. Yang, Cooperative adaptive fault-tolerant tracking control for a class of multi-agent systems with actuator failures and mismatched parameter uncertainties, IET Control Theory Appl. 9 (8) (2015) 1274-1284.

[23] Z. Gallehdari, N. Meskin, K. Khorasani, An $h_{\infty}$ cooperative fault recovery control of multi-agent systems, Automatica 84 (2017) $101-108$.

[24] G. Wang, R. Xue, C. Zhou, J. Gong, Complex-valued adaptive networks based on entropy estimation, Signal Processing 149 (2018) $124-134$.

[25] G. Wang, R. Xue, J. Zhao, Switching criterion for sub-and super-gaussian additive noise in adaptive filtering, Signal Processing 150 (2018) $166-170$.

[26] G. C. Goodwin, R. L. Leal, D. Q. Mayne, R. H. Middleton, Rapprochement between continuous and discrete model reference adaptive control, Automatica 22 (2) (1986) 199-207.

[27] H. Hu, B. Jiang, H. Yang, Reliable guaranteed-cost control of delta operator switched systems with actuator faults: mode-dependent average dwell-time approach, IET Control Theory Appl. 10 (1) (2015) 17-23.

[28] R. H. Middleton, G. C. Goodwin, Digital control and estimation: a unified approach, Prentice Hall Professional Technical Reference, 1990.

[29] H. Hu, B. Jiang, H. Yang, Robust fault-tolerant control for uncertain delta operator switched systems, IET Control Theory Appl. 8 (2) (2014) $120-130$.

[30] C. Qin, Z. Xiang, H. R. Karimi, Robust stability and stabilization of switched systems with time-varying delays using delta operator approach, Math. Prob. Eng. 2013 (1) (2013) 1-11.

[31] S. Chen, D. W. Ho, M. Liu, Consensus protocol for multiple delta operator systems, Syst. and Control Lett. 107 (2017) 1-8.

[32] R. A. Horn, R. A. Horn, C. R. Johnson, Matrix analysis, Cambridge university press, 1990.

[33] F. Jiang, L. Wang, Consensus seeking of high-order dynamic multi-agent systems with fixed and switching topologies, Int. J. Control 83 (2) (2010) 404-420.

[34] W. He, J. Cao, Consensus control for high-order multi-agent systems, IET Control Theory Appl. 5 (1) (2011) $231-238$.

[35] Y. Xin, Y. Li, X. Huang, Z. Cheng, Consensus of third-order nonlinear multi-agent systems, Neurocomputing 159 (2015) 84-89.

[36] X. Zhao, L. Zhang, P. Shi, M. Liu, Stability and stabilization of switched linear systems with mode-dependent average dwell time, IEEE 
Trans. Autom. Control 57 (7) (2012) 1809-1815.

[37] K. Premaratne, R. Salvi, N. Habib, J. LeGall, Delta-operator formulated discrete-time approximations of continuous-time systems, IEEE Trans. Autom. Control 39 (3) (1994) 581-585.

[38] L. Xie, Output feedback $h_{\infty}$ control of systems with parameter uncertainty, Int. J. control 63 (4) (1996) $741-750$.

[39] H. Li, J. Wang, H. Du, H. R. Karimi, Adaptive sliding mode control for takagi-sugeno fuzzy systems and its applications, IEEE Trans. Fuzzy Syst.doi:10.1109/TFUZZ.2017.2686357.

[40] Q. Zhou, H. Li, L. Wang, R. Lu, Prescribed performance observer-based adaptive fuzzy control for nonstrict-feedback stochastic nonlinear systems, IEEE Trans. System, Man, and Cyberneticsdoi : 10.1109/TSMC . 2017.2738155.

[41] Q. Zhou, L. Wang, C. Wu, H. Li, Adaptive fuzzy tracking control for a class of pure-feedback nonlinear systems with time-varying delay and unknown dead zone, Fuzzy Sets Syst. 329 (2017) 36-60.

[42] L. Wang, Z. Wang, T. Huang, G. Wei, An event-triggered approach to state estimation for a class of complex networks with mixed time delays and nonlinearities, IEEE Trans. Cybern. 46 (11) (2016) 2497-2508.

[43] S. Liu, Y. Song, G. Wei, D. Ding, Y. Liu, Event-triggered dynamic output feedback rmpc for polytopic systems with redundant channels: Input-to-state stability, J. Frankl. Inst. 354 (7) (2017) 2871-2892.

[44] L. Wang, Z. Wang, Q.-L. Han, G. Wei, Event-based variance-constrained $h_{\infty}$ filtering for stochastic parameter systems over sensor networks with successive missing measurements, IEEE Trans. Cybern. 48 (3) (2018) 1007-1017.

[45] L. Zou, Z. Wang, J. Hu, H. Gao, On $h_{\infty}$ finite-horizon filtering under stochastic protocol: Dealing with high-rate communication networks, IEEE Trans. Autom. Control 62 (9) (2017) 4884-4890.

[46] Z. Wang, D. Ding, H. Dong, H. Shu, $h_{\infty}$ consensus control for multi-agent systems with missing measurements: the finite-horizon case, Syst. and Control Lett. 62 (10) (2013) 827-836.

[47] L. Zou, Z. Wang, H. Gao, Set-membership filtering for time-varying systems with mixed time-delays under round-robin and weighted tryonce-discard protocols, Automatica 74 (2016) 341-348.

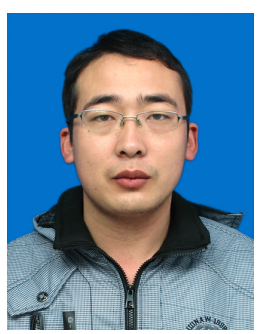

Dianhao Zheng received the B.Eng. degree in electronic science and technology from China University of Mining and Technology, Xuzhou, in 2009, and the M.S. degree in circuits and systems from University of Electronic Science and Technology of China, Chengdu, in 2012. He is currently working towards two Ph.D. degrees in circuits and systems at the University of Electronic Science and Technology of China and in engineering and information technology at the University of Technology Sydney. He was an engineer from 2012 to 2014. His research interests include cooperative control, multi-agent systems, and switched systems.

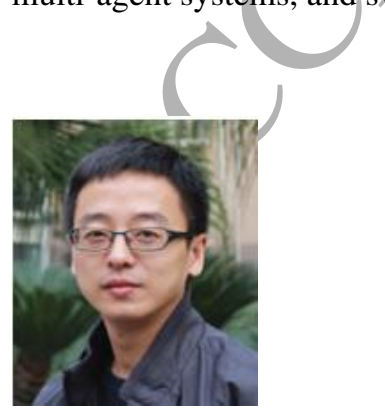

Hongbin Zhang received the B.Eng. degree in aero craft design from Northwestern Poly technical University, Xian, China, in 1999, and the M.Eng and Ph.D. degrees in circuits and systems from the University of Electronic Science and Technology of China, Chengdu, in 2002 and 2006, respectively. He has been with the University of Electronic Science and Technology of China, since 2002, where he is currently a professor. From August 2008 to August 2010, he has served as a research fellow with the Department of Manufacturing Engineering and Engineering Management, City Uni- 
versity of Hong Kong, Kowloon, Hong Kong. His current research interests include intelligent control, autonomous cooperative control and integrated navigation.

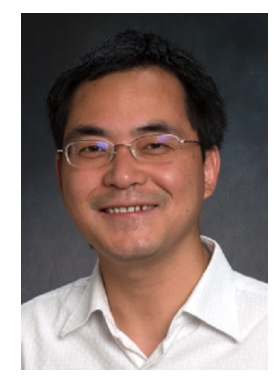

J. Andrew Zhang received the B.Sc. degree from Xi'an JiaoTong University, China, in 1996, the M.Sc. degree from Nanjing University of Posts and Telecommunications, China, in 1999, and the Ph.D. degree from the Australian National University, in 2004. Currently, Dr. Zhang is an associate Professor in School of Computing and Communications, University of Technology Sydney, Australia. He was a résearcher with Data 61, CSIRO, Australia from 2010 to 2016, the Networked Systems, NICTA, Australia from 2004 to 2010, and ZTE Corp, Nanjing, China from 1999 to 2001. Dr. Zhang's research interests are in the area of signal processing for wireless communications and sensing, and autonomous vehicular networks. He has won 4 best paper awards for his work. He is a recipient of CSIRO Chairman's Medal and the Australian Engineering Innovation Award in 2012 for exceptional research achievements in multi-gigabit wireless communications.

Gang Wang received the B.Eng. degree in Communication Engineering and the

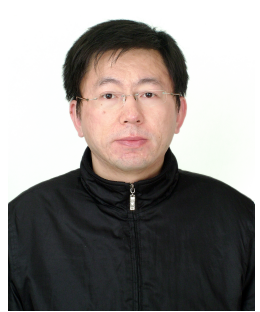
Ph.D.degree in Biomedical Engineering from University of Electronic Science and Technology of China,Chengdu, China, in 1999 and 2008, respectively. He is currently an Associate Professor with the University of Electronic Science and Technology of China. His current research interests include blind signal processing and intelligent systems. 